\title{
LA TÉRATOLOGIE DES TIQUES
}

\author{
Par Yvonne CAMPANA-ROUGET
}

\section{Gén éralités}

Historique. - Ce n'est que fort tard que l'on signale des anomalies chez les Tiques. C'est en effet seulement en 1899 que Neumann décrit les trois premiers cas tératologiques connus : la présence d'un œil surnuméraire chez un Hyalomma sp., l'absence de la première patte gauche chez un Amblyomma sp. et l'atrophie de la quatrième patte droite chez un Ixodes hexagonus. Chose curieuse, la première, et en même temps la plus intéressante de ces monstruosités, n'a été observée qu'une fois depuis.

Le premier monstre double n'est signalé qu'en 1909 par Warburton et Nuttall et le premier gynandromorphe en 1916 par Teresa Joan. Les documents que nous possédons ne remontent donc pas à plus de cinquante ans, alors qu'il existe des descriptions de monstruosités chez les Insectes depuis le début du $\mathrm{xix}^{\circ}$ siècle et chez les Crustacés depuis 1844. Même pendant cette courte période, les documents ne se succèdent pas à des intervalles très rapprochés : les publications les plus importantes sont celles de Warburton et Nuttall en 1909, de Nuttall en 1915, de P. Schulze en 1933-36-37, de E. Brumpt en 1934 et de Aboim-Inglez en 1942. En 1938, C. Desportes, à propos d'un monstre double, donne un tableau récapitulatif des diverses anomalies connues à cette époque. Il n'existe aucun ouvrage d'ordre plus général comme celui, par exemple, de Cappe de Baillon sur la tératologie des Insectes. Le remarquable livre de Bateson (1894), Materials for the study of Variation, englo-

(1) Le présent travail a fait l'objet d'une thèse de Sciences soutenue en 1950. Des circonstances diverses ne nous ayant pas permis de le publier jusqu'à présent, nous avons dû le remettre à jour. Or, durant ces dernières années, il a paru en Russie des travaux extrêmement importants qui doivent influer sur les conceptions actuelles de la biologie et de la tératologie des Tiques. Nous avons donc remanié tout le chapitre de la Tératologie naturelle et avons ajouté au chapitre de la Tératologie expérimentale les acquisitions nouvelles, mais nous n’avons pu transformer les résultats de notre propre expérimentation, dont certains passages paraîtront moins originaux qu'ils ne l'étaient au départ, de nouvelles expériences ayant été tentées depuis.

Ans. de Parasitologie, $\mathrm{t}$. XXXiV, $x^{\circ} 1-2,-1959$. 
bant les anomalies des Invertébrés, aussi bien que celles des Vertébrés, ne peut faire état d'aucun exemple chez les Tiques, puisqu'il n'y avait encore rien de signalé chez ces animaux.

Définition. - La tératologie est l'étude des monstres. Cette étude, pour être complète, doit comprendre, outre une partie descriptive, la biologie de l'animal monstrueux, l'évolution de l'anomalie et son déterminisme.

La notion de monstre est difficile à délimiter exactement; elle a d'ailleurs souvent varié au cours des années et selon les auteurs. Nous considérerons comme monstrueux, ainsi que le fait Balazuc, tout exemplaire non conforme à la structure habituelle du genre auquel il appartient, étant mises à part les mutilations survenues au cours du stade actuel. Cela ne nous empêchera pas d'établir une gradation des monstruosités, nous rapprochant ainsi de la classification de Geoffroy Saint-Hilaire, qui s'étend de la simple variété à la monstruosité vraie, par ordre de gravité et de complexité. Nous ne réserverons pas le terme de monstruosités aux variations d'origine embryonnaire, car ce serait méconnaitre l'action des agents extérieurs qui, comme nous le verrons plus loin, jouent un rôle fort important.

Classification. - Nous ne reviendrons pas sur les diverses classifications des monstruosités, qui sont bien connues et ont été résumées par J. Balazuc (1948). Elles sont d'ordre anatomique, tératologique ou étiologique et sont forcément toutes plus ou moins arbiraires, car des monstruosités d'aspect très différent peuvent avoir une cause commune et, inversement, des anomalies semblant très proches les unes des autres peuvent avoir des causes extrêmement diverses.

Nous adopterons l'ordre suivant :

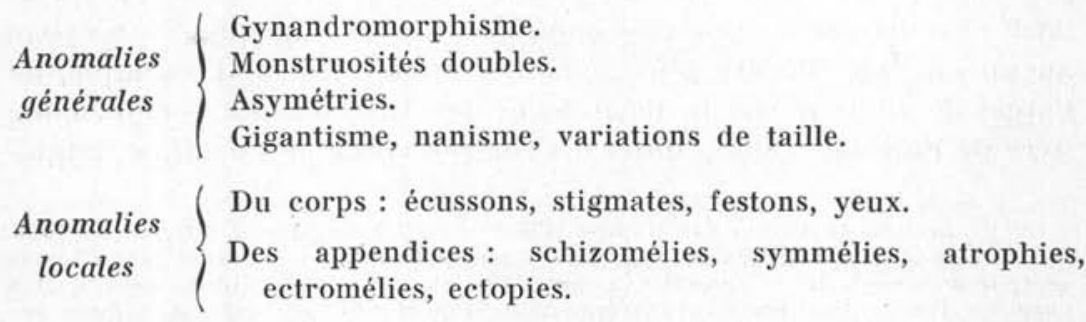

Les anomalies locales du corps portent soit sur l'ornementation, soit sur des organes particuliers, tels que les stigmates, dont la forme ou la position changent. 
Les anomalies du développement, telles que la prothétẻlie, par exemple, où apparaissent déjà chez les nymphes un certain nombre de caractères adultes, ne peuvent guère se rencontrer chez les Tiques : en effet, chez les Argasidés, le stade adulte ne se manifeste que par l'apparition de l'orifice génital ; chez les Ixodidés, les caractères mâles sont marqués par la présence d'écussons dorsaux et parfois ventraux. Il n'est donc pas impossible que de telles formations apparaissent prématurément chez la nymphe, mais aucun cas n'a jamais été signalé.

Les anomalies des appendices sont à peu près les mêmes que chez les Insectes, mais moins nombreuses et moins variées : c'est ainsi que l'on n'a jamais décrit d'hypermélie, c'est-à-dire d'augmentation linéaire du nombre des articles.

Fréquence. - L'historique de ce travail nous a déjà fait entrevoir que les anomalies étaient peu nombreuses chez les Tiques. Tous les auteurs décrivant des monstruosités chez ces animaux commencent par en faire remarquer la rareté. Voici ce que certains d'entre eux écrivent à ce sujet :

- Neumann (1899) : "Il est rare de rencontrer des anomalies chez les Acariens. Je ne crois pas qu'on en ait jamais signalé chez les Ixodidés. A l'occasion d'une révision que j'ai faite de cette famille, j'ai dû examiner des milliers de spécimens. Trois d'entre eux s'écartaient de leur type spécifique par une anomalie réelle. »

- Warburton et Nuttall (1909) : "The abnormalities which we have encountered as the result of examining a vast material are usually slight and infrequent. »

- Sénevet (1922) : «Au cours d'une recherche portant sur près de 3.000 Tiques d'espèces diverses, il m'a été donné de rencontrer deux cas de monstruosités. »

- E. Brumpt (1934) : «Les monstruosités sont très rares chez les Ixodidés et seuls les représentants de la sous-famille des Ixodinés en ont offert un certain nombre de cas. En ce qui concerne mes recherches personnelles, en dehors de deux exemplaires gynandromorphes trouvés dans la nature, je n'ai rencontré qu'un seul exemplaire du même type et six cas de monstruosités chez des individus en partie doubles sur des dizaines de milliers de larves hexapodes et sur des milliers de nymphes ou d'adultes élevés au laboratoire. Je partage done l'opinion de Neumann, de Nuttall et de ses collaborateurs, de Sénevet, qui, n'ayant vu que quelques anomalies au cours de leurs études portant sur des milliers d'exemplaires d'Ixodinés divers, concluent à leur extrême rareté dans ce groupe d'Acariens. » 
- Delpy (1936) : «Depuis quatre ans, nous examinons journellement de nombreux Ixodidæ et en particulier des Hyalomma envoyés de diverses parties de l'Iran. Parmi des milliers de Tiques, nous n'avons trouvé que trois cas de monstruosité caractérisée. Par contre, la pratique de mensurations précises nous a montré que des asymétries plus ou moins accusées, portant sur les organes chitineux pairs, sont fréquentes. »

Nous constatons donc, en résumé, en dehors des cas expérimentaux, une relative fréquence des anomalies légères telles que l'asymétrie des écussons, la fusion des festons, etc..., par contre, une grande rareté des monstruosités importantes. La répartition de ces dernières est assez curieuse : les gynandromorphes viennent en tête, suivis par les monstres doubles. Mais les anomalies d'appendices, ectromélies et surtout schizomélies, sont très rares. Chez les Coléoptères, par exemple, ce sont les anomalies d'appendices qui sont les plus nombreuses, ce qui parait plus conforme à nos connaissances sur la tératologie. En effet, les monstruosités d'origine embryonnaire, sauf si elles sont dues à des mutations, sont en principe très peu fréquentes ; par contre, les malformations d'appendices, généralement dues à des causes extérieures, devraient se rencontrer plus souvent. Nous avons recherché la cause de cette contradiction, et croyons qu'elle est plus apparente que réelle.

Les Insectes ont été récoltés de tout temps par des collectionneurs, scientifiques ou amateurs, qui ont ainsi amassé des quantités de documents sur des animaux prélevés directement dans la nature; la proportion de monstruosités décrites doit done être à peu près celle qui existe réellement. Il en est tout autrement pour les Tiques qui intéressent surtout les parasitologistes par les maladies qu'elles sont susceptibles de transmettre. La récolte de quelques exemplaires suffit, le plus souvent, pour assurer un élevage important, puisqu'une seule femelle pond en une fois plusieurs milliers d'œufs. Aussi le nombre de Tiques récoltées dans la nature, bien que déjà considérable, est-il bien inférieur à celui des Tiques d'élevage. Celles-ci sont soustraites en grande partie aux influences extérieures qui peuvent déterminer des monstruosités.

L'action du milieu n'est sans doute pas très grande sur les monstruosités d'origine embryonnaire, dont le pourcentage doit rester à peu près constant, mais elle est très importante pour les anomalies causées par des agents extérieurs. Ceux-ci ne pouvant plus intervenir, la proportion de ces anomalies doit être beaucoup moins élevée dans un élevage que dans la nature. D'autres facteurs peuvent d'ailleurs entrer en jeu pour provoquer de nouvelles monstruo- 
sitís, par exemple l'influence d'un milieu confiné. Cela a été démontré pour certains Insectes, mais ne parait pas intervenir dans la genèse des anomalies connues chez les Tiques.

Etiologie. - Nous étudierons avec chaque groupe d'anomalies leurs causes probables. Il nous suffit de savoir pour l'instant qu'elles se ramènent à quatre :

$1^{\circ}$ les mutations proprement dites, faisant varier un caractère héréditaire ;

2" les mutations somatiques, qui ont sans doute même origine, mais ont pour siège une cellule somatique et ne sont pas héréditaires ;

3 " la formation d'embryons en partie doubles, provenant d'œufs composés ou d'un accident embryonnaire plus tardif ;

$4^{\circ}$ les agents extérieurs qui peuvent être mécaniques, physiques ou chimiques et interviennent à un stade quelconque du développement.

\section{PREMIÊRE PARTIE \\ TÉRATOLOGIE NATURELLE \\ Anomalies générales}

\section{Gynandromorphisme}

Parmi les animaux à dimorphisme sexuel suffisamment marqué pour que l'on puisse découvrir des gynandromorphes, la proportion de tels monstres est très variable suivant les groupes envisagés : chez les Insectes, par exemple, les gynandromorphes sont beaucoup plus répandus chez les Lépidoptères ou les Diptères que chez les Coléoptères, où l'on ne connait qu'une vingtaine de cas.

Nous ne pouvons espérer trouver de tels cas chez les Argasidæ, dont seule diffère la forme de l'orifice génital selon le sexe. Il n'en est pas de mème chez les Ixodidæx, dont nous allons tout d'abord rappeler les principaux caractères liés au sexe :

$1^{\circ}$ La taille de l'hypostome et la disposition des dents varient d'un sexe à l'autre chez certaines espèces (Ixodes).

$2^{\circ}$ La base du capitulum présente sur la face dorsale deux aires poreuses, orgaines sensoriels, chez la femelle uniquement. 
$3^{\circ}$ Le scutum de la femelle, triangulaire, ne dépasse jamais le tiers antérieur du corps. Celui du mâle recouvre toute la face dorsale.

$4^{\circ}$ Les foveæ dorsales, autres organes sensoriels, se trouvent au milieu du scutum mâle, en arrière du scutum femelle.

$5^{\circ}$ Les épines des coxa sont souvent plus développées chez le mâle.

$6^{\circ}$ L'orifice génital est arrondi ou allongé transversalement chez la femelle, il est recouvert d'une petite plaque chitineuse chez le mâle.

$7^{\circ}$ Les stigmates respiratoires peuvent être de forme différente suivant le sexe (Rhipicephalus, Hyalomma, etc.).

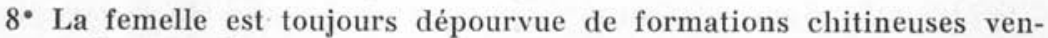
trales, que l'on rencontre dans beaucoup de genres chez le mâle, sous forme de plaques, d'écussons ou de pointes de formes variables.

Ces écussons portent des noms différents suivant les auteurs. Pour éviter les confusions, nous adoptons la nomenclature suivante :

Ecussons anaux bordant l'anus à gauche et à droite.

Ecussons adanaux situés à droite et à gauche des écussons anaux.

Ecussons subanaux, situés au-dessous des écussons anaux.

Jusqu'en 1948, les cas de gynandromorphisme sont très rares, puisqu'ils ne dépassent pas 20, depuis la découverte du premier en 1916 (publié en 1919) par Teresa Joan. Brusquement, au cours des dix dernières années, on voit apparaître un nombre relativement considérable de tels monstres, soit isolément, au cours de récoltes (Santos Dias) ou d'études de collections (Pavlovsky et Bernadskaia), soit par lots d'importance variable, dans des élevages (Pervomaisky).

Si les cas isolés que nous venons de citer apparaissent comme tout aussi fortuits que ceux de la première période, il n'en est pas de même pour les gynandromorphes de Pervomaisky ; il s'agit ici de tératologie expérimentale : en effet, ce dernier, au cours de longues et minutieuses expériences sur des populations de Hyalomma, puis de Rhipicephalus de diverses espèces, se livre à des croisements inter-espèces, et c'est parmi les hybrides issus de ces croisements qu'il découvre la plus grande partie des monstres qu'il décrit. En 1950, sur 72 gynandromorphes, 63 sont des hybrides de Hyalomma anatolicum excavatum et de Hyalomma marginatum marginatum, les neuf autres sont des souches pures des mêmes espèces. Sur sept populations d'hybrides obtenus de la même façon, cinq comprenant de 218 à 478 spécimens chacune n'ont révélé aucun gynandromorphe, une en a présenté 2 sur 148 spécimens et une a comporté 61 gynandromorphes sur 435 Tiques. De nouveaux croi- 
sements Rhipicephalus bursa $\times R$. sanguineus portent en 1954 le nombre des gynandromorphes de Pervomaisky à 113, dont 91 hybrides. Nous reviendrons plus loin sur l'intérêt que présente cette découverte au point de vue génétique. L’auteur considère le gynandromorphisme comme la limite extrême de la variabilité qu'il observe chez ses hybrides ; il trouve en effet nombre d'autres anomalies et des variations de la taille des écussons anaux chez les mâles.

Un deuxième fait important est l'observation de tels animaux vivants, qui a permis l'étude du gorgement, du comportement sexuel et de la fécondité, rarement ou incomplètement connus jusque-là.

Enfin, la comparaison d'un aussi grand nombre d'individus a permis à l'auteur de donner une classification plus complète et plus précise que celles qui ont pu être proposées antérieurement.

Pavlovsky et Bernadskaia (1948) classent les gynandromorphes selon quatre types : gynandromorphes bi-partis, bi-partis associés à une mosaïque, mosaïques pures, gynandromorphes partiels, ces derniers comportant les spécimens où l'un des deux sexes prédomine considérablement, jusqu'au point où l'on ne retrouve plus que des vestiges de l'autre sexe.

Pervomaisky donne le tableau suivant :

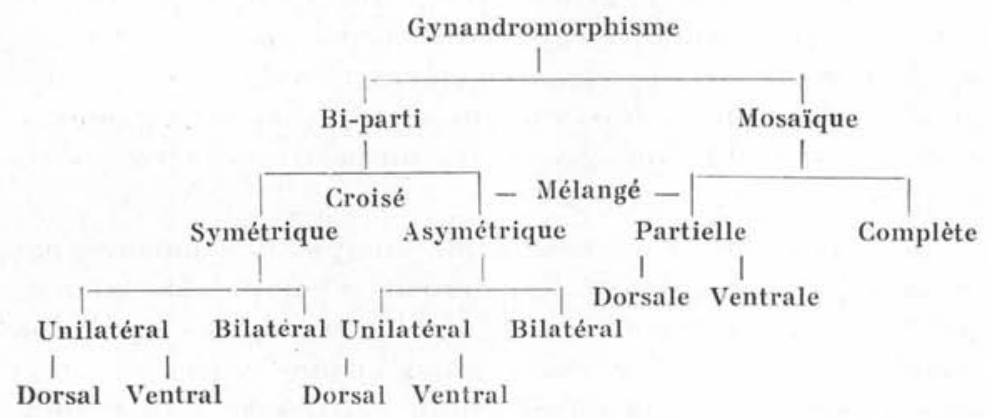

Le terme de "mélangé 》 est la traduction littérale, nous lui préférons celui de gynandromorphisme intriqué, comprenant les spécimens bi-partis qui présentent en outre des ilots de chitine en mosaïque dans leur moitié mâle ou femelle (mâle le plus souvent). Chez les bi-partis symétriques (par rapport à l'axe médian du corps), la division peut intéresser les deux faces et, dans ce cas, toute une moitié du corps est du même sexe ou, au contraire, l'anomalie est croisée, c'est-à-dire qu'à la moitié dorsale mâle corres- 
pond une moitié ventrale femelle et inversement; ce dernier cas semble assez rare (fig. 23).

Le terme de bi-parti asymétrique englobe ici tout ce qui n'est pas strictement moitié mâle et moitié femelle C'est, à notre avis, une subdivision un peu large, car elle comprend des individus d'un aspect fort différent, depuis le gynandromorphe antéro-postérieur de Lengerken, c'est-à-dire un individu dont un quart du corps est du sexe opposé à l'ensemble, jusqu'à des spécimens qui paraissent à première vue unisexués, et dont seul un examen attentif révèle qu'il y a un vestige du caractère sexuel opposé. Le premier cas représente un fait précis, l'anomalie survenant lors de la deuxième division de l'œuf, alors que, dans le second cas, tous les degrés peuvent exister, et il devient impossible de savoir à quel stade de division le gynandromorphisme a fait son apparition.

Les dénominations généralement adoptées pour désigner les différents gynandromorphes sont en réalité peu satisfaisantes : celle de mi-parti ou bi-parti, préférable à « symétrie bilatérale » employée par Lengerken, est assez bonne pour les spécimens moitié mâle, moitié femelle, mais devient assez choquante quand elle désigne des individus extrêmement asymétriques.

En examinant de près les gynandromorphes cités dans la littérature, on s'aperçoit que leur classification selon les normes établies est assez difficile à réaliser, car ils correspondent rarement complètement au type théorique : tel gynandromorphe mi-parti symétrique possède une aire poreuse plus ou moins réduite du côté mâle, tel autre a des écussons réduits du côté mâle, et on ne sait plus si on doit classer ces individus parmi les mi-partis symétriques ou asymétriques.

Une telle classification ne peut tenir compte des phénomènes d'induction qui se produisent fréquemment, d'autant plus intensément que la prédominance d'un sexe sur l'autre est plus nette : on a alors une féminisation des plages mâles ou une masculinisation des plages femelles qui rendent difficile le classement de tels individus uniquement d'après la morphologie externe.

Aussi proposons-nous une nouvelle classification basée sur le stade de division embryonnaire auquel survient l'anomalie ; quand elle apparaît à la première division de l'œuf, le monstre est un miparti parfait, mais peut cependant présenter des plages induites : nous l'appellerons « protogynandre ».

Lorsque l'anomalie se produit seulement à la deuxième division de l'œuf, elle aboutit à ce que Lengerken appelait un gynandromor- 
phe antéro-postérieur, dont un quadrant seulement est de sexe opposé au reste du corps : nous l'appellerons «deutérogynandre».

Enfin, lorsque l'anomalie se produit plus tard au cours du développement embryonnaire, il n'y a plus que des plages parfois extrêmement réduites d'un des sexes ; il devient alors impossible de préjuger du stade auquel s'est produit le dysfonctionnement: ces individus, qui correspondent à peu près aux gynandromorphes partiels de Pavlosky, prendront le nom de «métagynandres».

Nous ne changeons rien au reste de la classification, le gynandromorphisme pouvant être bilatéral ou unilatéral, et, dans ce cas, dorsal ou ventral. Les mosaïques et le gynandromorphisme intriqué ne subissent pas non plus de changements, étant sous-entendu que l'intrication peut se produire aussi bien sur des proto- que sur des deutéro- ou des métagynandres.

Nous allons maintenant décrire un gynandromorphe nouveau récolté par J. Théodoridès, dans les environs de Banyuls, en 1950.

\section{Hyalomma excavatum Koch 1844 (fig. 11)}

Exemplaire gorgé à forte prédominance femelle. Le bord postérieur est resserré à droite par une plaque de chitine dure, irrégulière et de couleur sombre. La face ventrale montre une plage mâle bien délimitée, allant de l'anus au bord postérieur et n'atteignant pas le bord droit du corps : en effet, le stigmate est de type femelle comme le stigmate gauche. La partie mâle comporte trois écussons typiques et normalement développés et trois festons. Il ne s'agit plus d'un gynandromorphisme apparu à la deuxième division; dans ce cas, le dimorphisme aurait atteint le stigmate ; la délimitation encore très nette permet de penser qu'il est apparu à la troisième division de l'œuf : nous le classerons done parmi les métagynandres.

Cet intéressant spécimen a pondu au bout d'un mois environ. La ponte, relativement peu importante, a presque entièrement éclos et nous avons pu obtenir 295 adultes, comprenant à peu près le même nombre de mâles et de femelles. Aucun d'entre eux n'a présenté d'anomalies.

Nous donnons ci-dessous la liste des différents gynandromorphes connus, classés autant que possible selon l'importance de leur anomalie. Nous ne les redécrivons pas, mais donnons les détails intéressants au point de vue morphologique ou biologique pour certains d'entre eux. 


\section{GYNANDROMORPHES.}

\section{Protogynandres :}

Amblyomma cajennense Fabr. 1787. Brésil. Exemplaire gorgé décrit par da Fonseca en 1935.

Cet exemplaire est apparemment le type parfait du protogynandre (fig. 1).

Amblyomma dissimile Koch 1844. Tique d'élevage, à jeun. Brumpt, 1934

(fig. 2).

Il existe deux aires poreuses, mais celle du còté mâle est nettement plus petite que l'autre. La deuxième patte droite (côté mâle) porte un appendice surnuméraire, dont nous aurons l'occasion de reparler (cf. schizomélies).

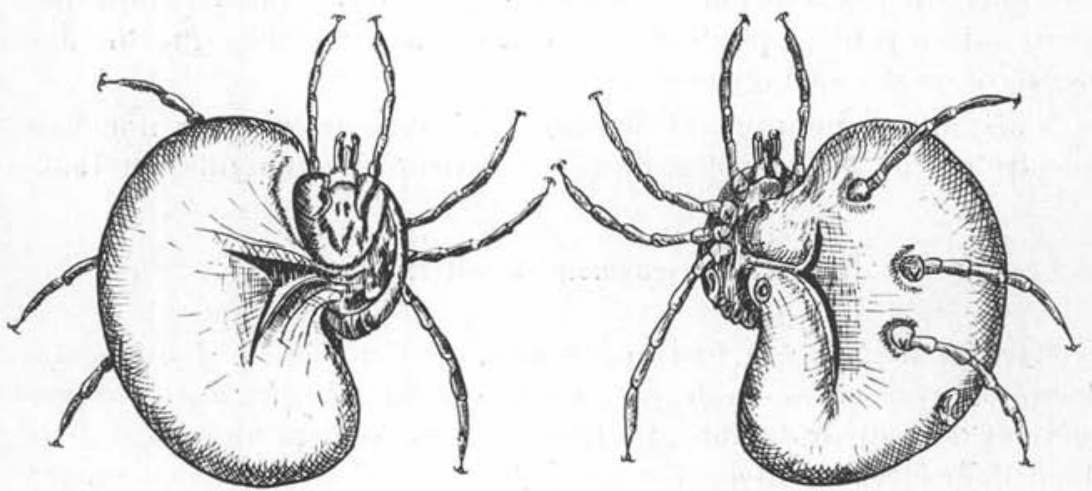

Fig. 1. - Amblyomma cajennense.

Gynandromorphe exactement mi-parti. (D'après F. da Fonseca).

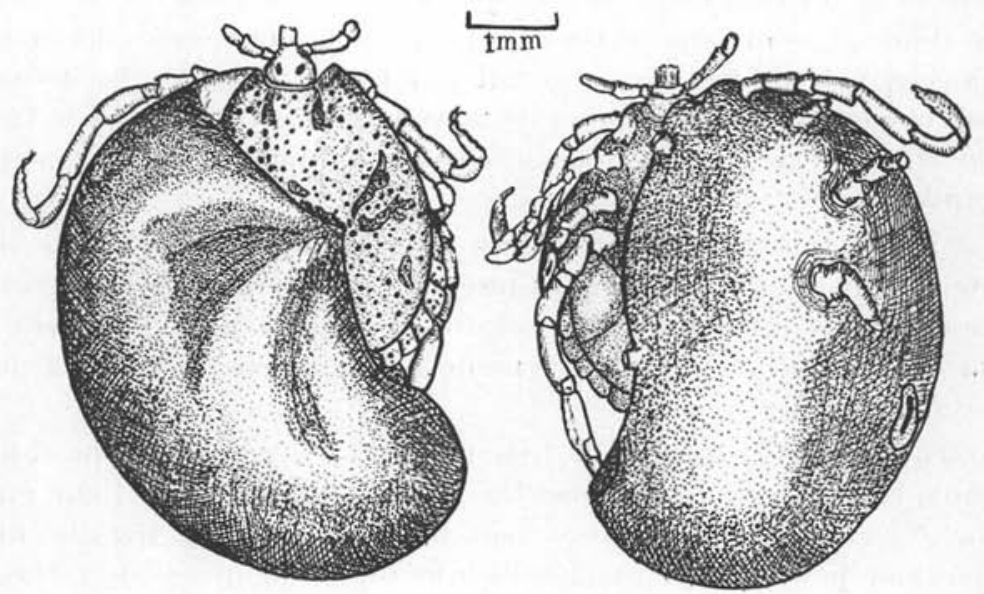

Fıg. 2. - Amblyomma dissimile. Protogynandre à légère prédominance femelle (présence de deux aires poreuses inégales). Noter la bifurcation de la deuxième patte droite. (D'après E. Brumpt). 
L'auteur met l'animal à jeun avec deux mâles gorgés et observe un accouplement ; il le met ensuite en présence de trois femelles en diapause virginale (à demi-gorgées), et n'observe aucune tentative de copulation ; l'animal se comporterait donc comme une femelle (l'orifice génital est d'ailleurs femelle); cependant la dernière expérience n'est pas très concluante, car les mâles d'A. dissimile n'ont pas d'appétit sexuel lorsqu'ils sont à jeun.

L'animal est ensuite placé avec plusieurs mâles sur une tortue, sur laquelle il se fixe et reste pendant sept semaines, puis il meurt, presque complètement gorgé, sans avoir été trouvé en copulation. Il n'a pas été
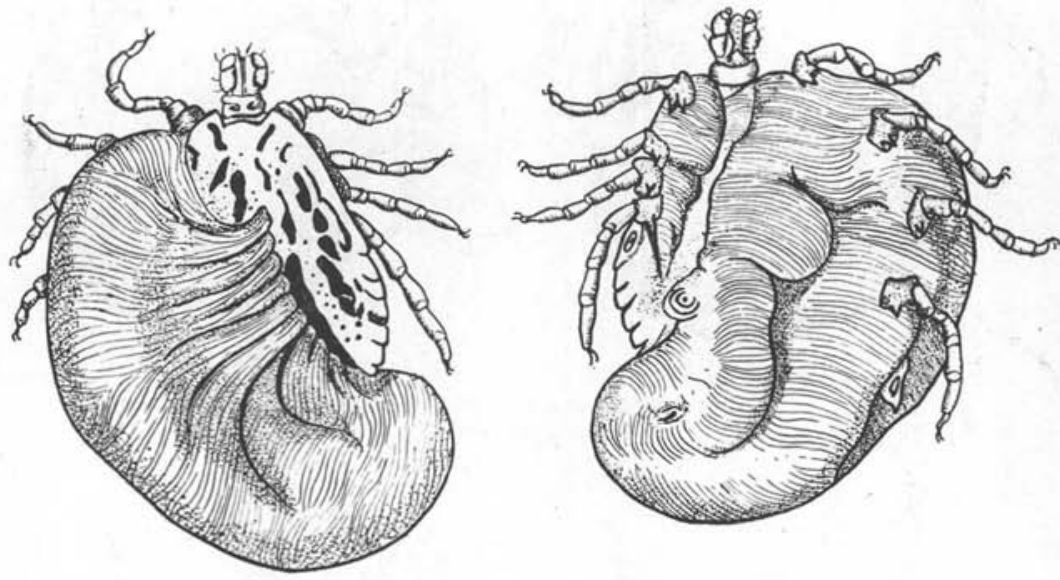

Fig. 3. - Amblyomma neumanni. Protogynandre

á nette prédominance femelle antérieurement. (D'après T. Joan).

fait de dissection de l'appareil génital à ce moment; nous avons tenté d'en faire une quinze ans après, mais n'avons pu discerner qu'un fragment d'oviducte.

A. neumanni Ribaga 1902. Argentine. Exemplaire gorgé décrit par Teresa Joan en 1916.

Antérieurement, il existe une prédominance femelle, avec deux aires poreuses identiques (fig. 3).

Amblyomma variegatum Fabr. 1794. Dahomey. Brumpt, 1922 et 1934.

Le capitulum manque, le reste du corps est exactement mi-parti (fig. 4).

Hyalomma detritum Schulze. Russie. Pavlovsky, 1940.

Il semble exister une aire poreuse petite et mal délimitée du côté mâle ; l'orifice génital est divisé en deux moitiés mâle et femelle (le plus souvent, cet orifice est mâle ou femelle) (fig. 5).

Hyalomma detritum Sch. Russie. Pavlovsky et Bernadskaia, 1948. 


\section{Hyalomma dromedarii Koch. Schulze 1950.}

Exemplaire assez exactement mi-parti. (Non figuré par l'auteur). Cette tique provient d'un élevage où sont apparus plusieurs gynandromorphes (deux seulement sont décrits).

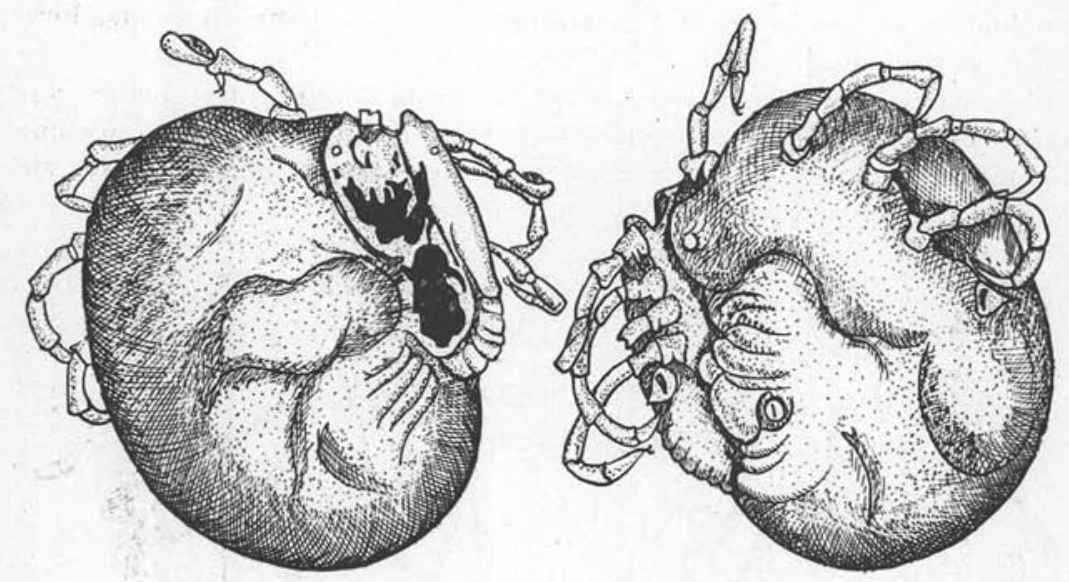

F16. 4. - Amblyomma variegatum. Le capitulum manque. Protogynandre presque parfait. (D'après E. Brumpt).
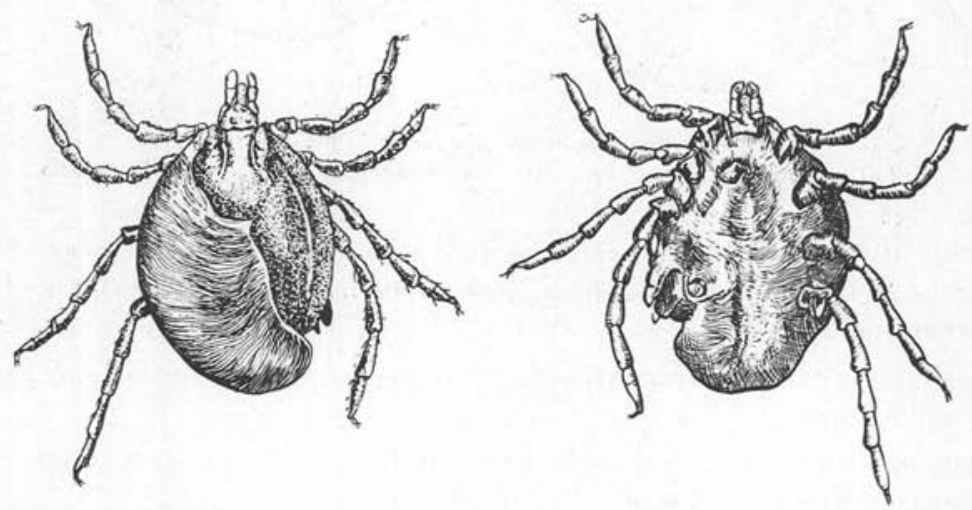

Fu. 5. - Hyalomma detritum. Exemplaire presque exactement mi-parti. Les écussons ventraux fortement rejetés vers la droite sont visibles en vue dorsale. (D'après E. Pavlovsky).

Hyalomma marginatum balcanicum P. Sch. et Schl. Macédoine. Exemplaire à demi-gorgé décrit par Schulze en 1933 (fig. 6, A).

La partie antérieure du corps est entièrement mâle, la division n'apparaissant qu'au début du scutum sur la face dorsale et à la deuxième paire de pattes sur la face ventrale. L'appareil génital est entièrement 
femelle-avec un ovaire bien développé, alors que l'orifice génital est mâle.

Malgré une division irrégulière, ce spécimen possède donc à peu près une moitié mâle et une moitié femelle.

Hyalomma marginatum Sch. Russie. Pavlovsky et Bernadskaia 1948.

Rhipicephalus turanicus Power. Russie. Pavlovsky 1940.

L'écusson femelle est mal délimité (fig. 7).
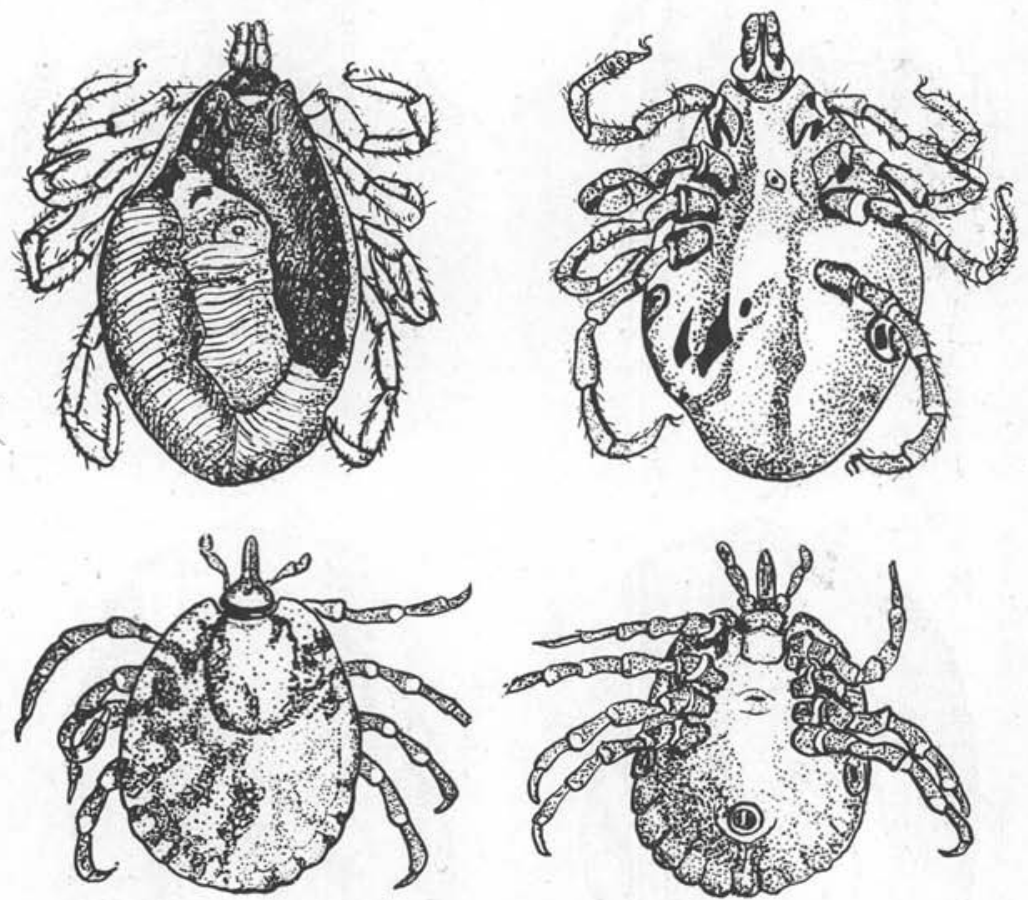

F1G. 6. - En haut, Hyalomma marginatum balcanicum.

En bas, Amblyomma scutatum. Protogynandre intriqué. (D'après P. Schulze).

D'après Pervomaisky, il faut ajouter à cette liste les deux exemplaires suivants :

Hyalomma marginatum turanicum et Hyalomma asiaticum asiaticum Russie. Tselitcheva 1948.

Pervomaisky les classe en effet parmi les bi-partis bilatéraux (nous n'avons malheureusement pas pu nous procurer ce travail).

\section{Deutérogynandres :}

Rhipicephalus bursa Canestrini et Fanz, 1877. Portugal. Exemplaire gorgé décrit par E. Brumpt en 1927 et 1934.

Le quadrant mâle est situé antérieurement, comprenant la moitié du capitulum et les quatre pattes droites (fig. 8). 


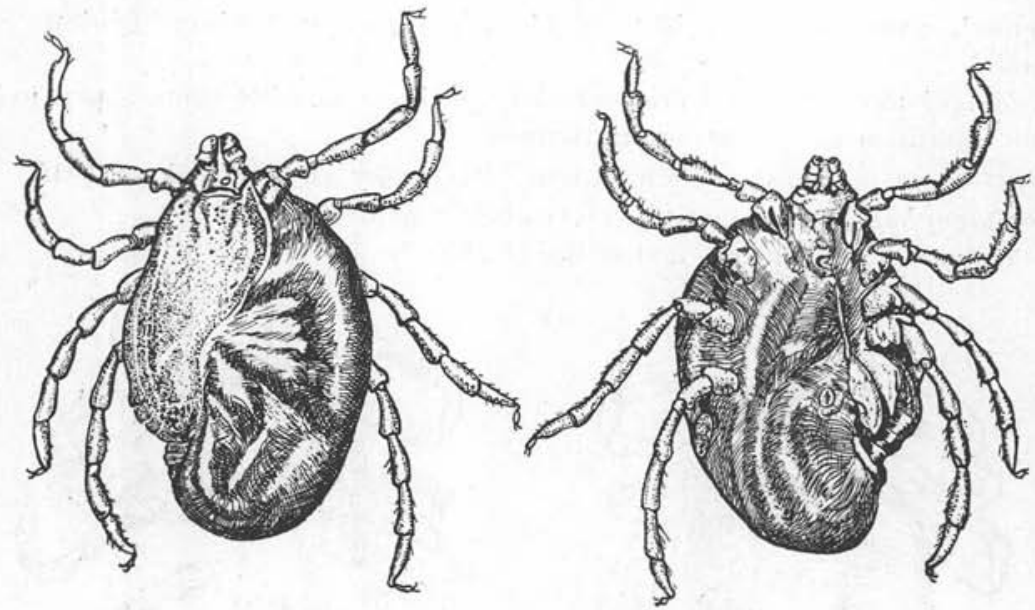

FIG, 7. - Rhipicephalus turanicus. Légère prédominance femelle du côté ventral. (D'après E. Pavlovsky).

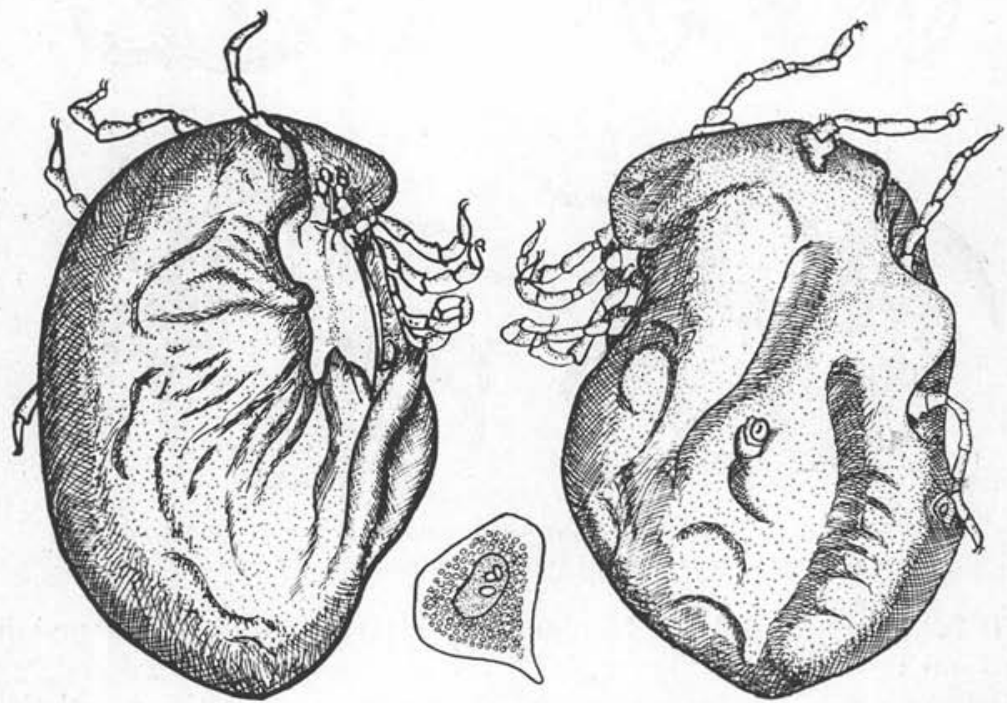

FIG. 8. - Rhipicephalus bursa. Au centre, stigmate gauche très agrandi. A gauche, vue ventrale : la tête et une partie du côté mâle sont masquées par la chitine femelle particulièrement hypertrophique. (D’après E. Brumpt).

Rhipicephalus naevei Warburton, 1912. Mozambique. Travassos Santos Dias, 1951 (fig. 9, A et B).

Rhipicephalus simus Koch, 1844. Mozambique. Travassos Santos Dias, 1953 (fig. 9, D).

Chez ces deux derniers, le quadrant mâle est postérieur. 

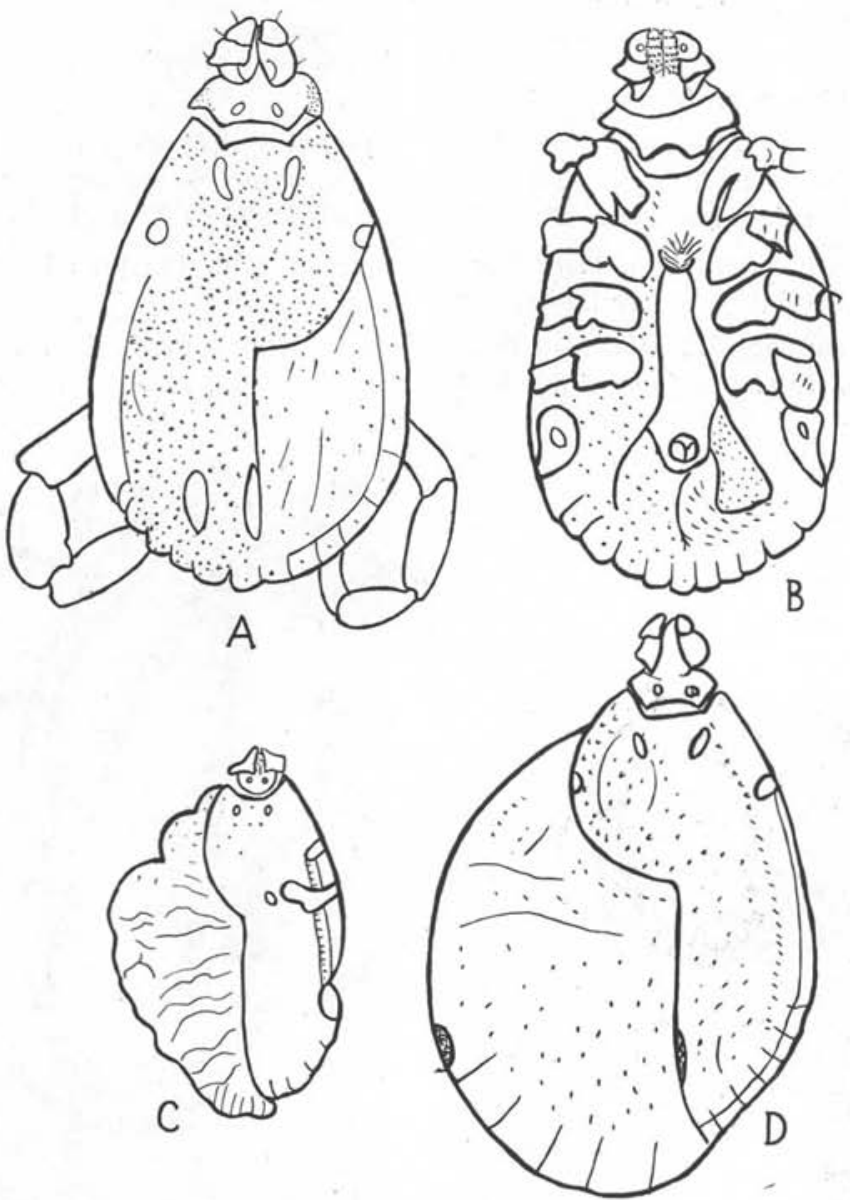

FIG. 9. - A et B, Rhipicephalus naevi. Deutérogynandre; C, Haemaphysalis leachii. Deutérogynandre intriqué ; D, Rhipicephalus simus. Deutérogynandre. (D'après T. Santos Dias).

\section{Métagynandres :}

Hyalomma isaaci Sharif 1928. Indes. Exemplaire gorgé décrit par Sharif en 1930.

La région mâle est seulement représentée par une étroite bande de chitine partant de la pointe du scutum et se dirigeant vers le bord postérieur qu'elle n'atteint pas (fig. 10).

Hyalomma excavatum Koch.

Exemplaire décrit précédemment dans ce travail (fig. 11). 


\section{Gynandromorphes intriqués :}

Amblyomma scutatum Neumann 1899. Autriche. Exemplaire à jeun décrit par Schulze en 1933.

Il s'agit d'un protogynandre chez lequel des îlots de chitine femelle apparaissent dans le scutum mâle. On remarque la présence de trois foveæ dorsales, une mâle à gauche, et deux femelles à droite (fig. 6, B).

Hyalomma detritum damascenium Schulze. et Schl. Turquie. Exemplaire gorgé décrit par Schulze en 1936.

Deutérogynandre montrant des îlots de chitine femelle dans la région mâle ventrale. Les fovæ dorsales sont décalées (fig. 12).

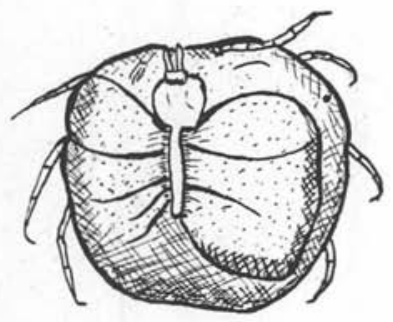

Fig. 10. - Hyalomma isaaci. Métagynandre. (D’apr. M. Sharif).

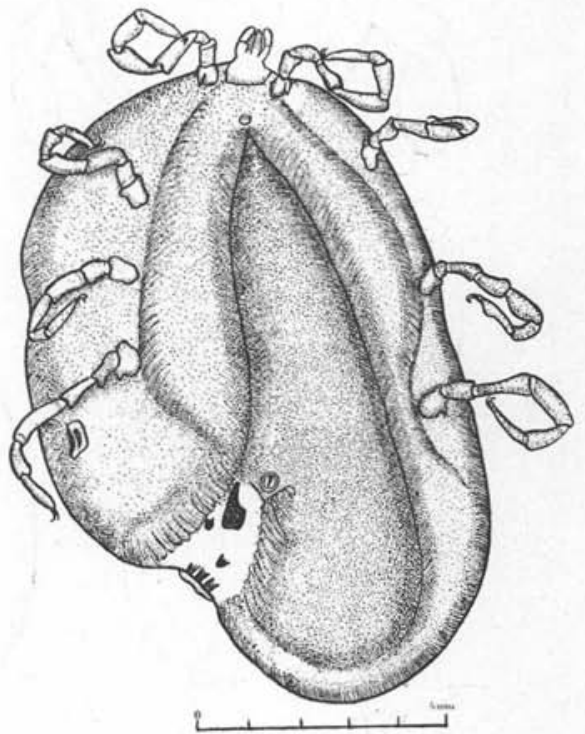

FiG. 11. - Hyalomma excavatum. Deutérogynandre. Original.

Haemaphysalis leachii leachii (Audouin, 1826). Mozambique. T. Santos Dias, 1952.

Deutérogynandre présentant quelques îlots de chitine femelle sur la région dorsale mâle (fig. $9, \mathrm{C}$ ).

Hyalomma dromedarii Koch, Schulze 1950.

Exemplaire à prédominance femelle, présentant à droite un scutum mâle irrégulier et, sur la face ventrale du même côté, un stigmate mâle et une série d'écussons qui paraissent de taille réduite. Nous le rangerons parmi les métagynandres intriqués (mosaïque dorsale, un quart postérieur mâle). 

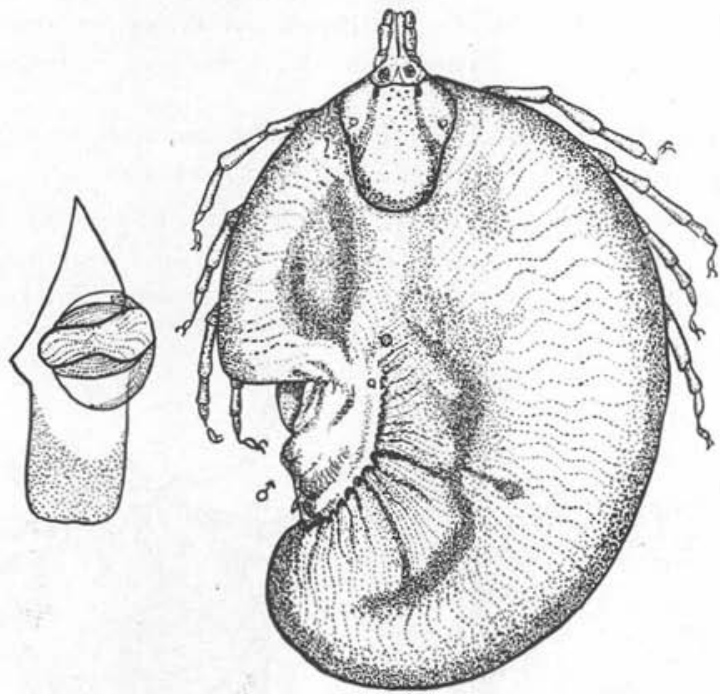

FiG. 12. - Hyalomma detritum damascenium. Deutérogynandre intriqué. Noter le décalage des fovex dorsales. A gauche, écusson anal montrant une enclave de chitine femelle. (D'après P. Schulze).
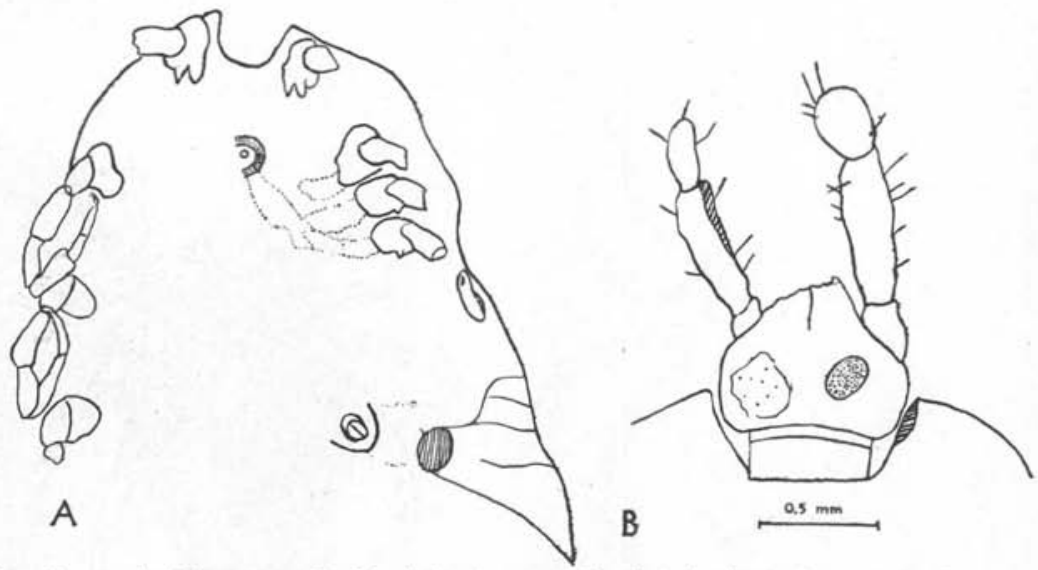

Fig. 13. - Amblyomma tholloni. Protogynandre intriqué. A, face ventrale montrant l'orifice génital hermaphrodite; $B$, tête présentant une seule aire poreuse bien limitée.

Amblyomma tholloni Neumann 1899, T. Santos Dias 1948. Mozambique. L'auteur ne place pas cet exemplaire parmi les gynandromorphes et pense que les anomalies citées découlent d'une hyperchitinisation. Or, sa description nous montre que la moitié droite du corps est entièrement femelle, alors que la moitié gauche présente les caractères suivants: 
absence d'aire poreuse, plaques chitineuses dorsales, vestiges de scutum mâle, chitinisation de la région ventrale, présence de festons, stigmate mâle.

Tous ces caractères nous indiquent que nous, nous trouvons en présence d'un protogynandre intriqué (fig. 13, A et B).

Rhipicephalus bursa Can. et Fanz. 1877. Russie. Pavlovsky 1940.

La face dorsale est mi-partie, la face ventrale forme une mosaïque, donnant un aspect très irrégulier au bord postérieur (fig. 14).

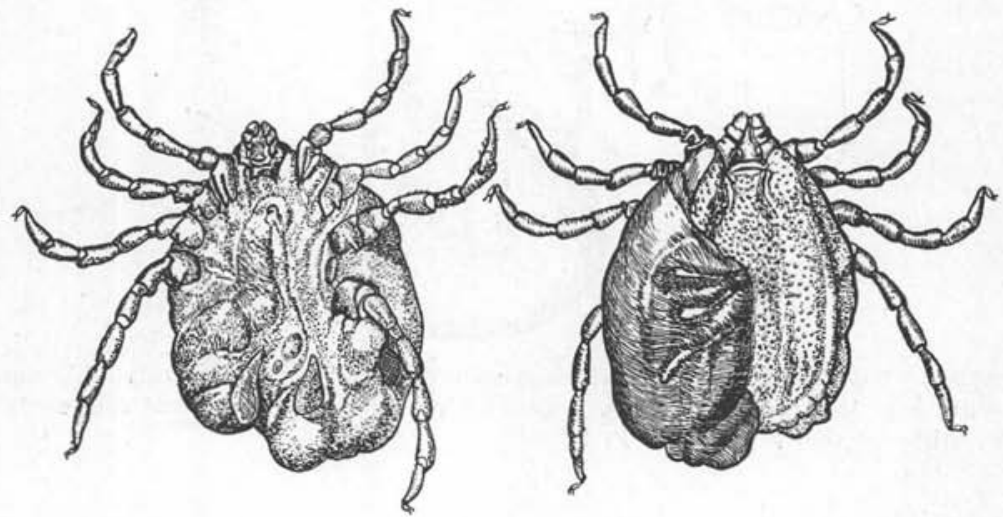

FIG. 14. - Rhipicephalus bursa. Face dorsale mi-partie. Mosaïque ventrale. (D'après E. Pavlovsky).
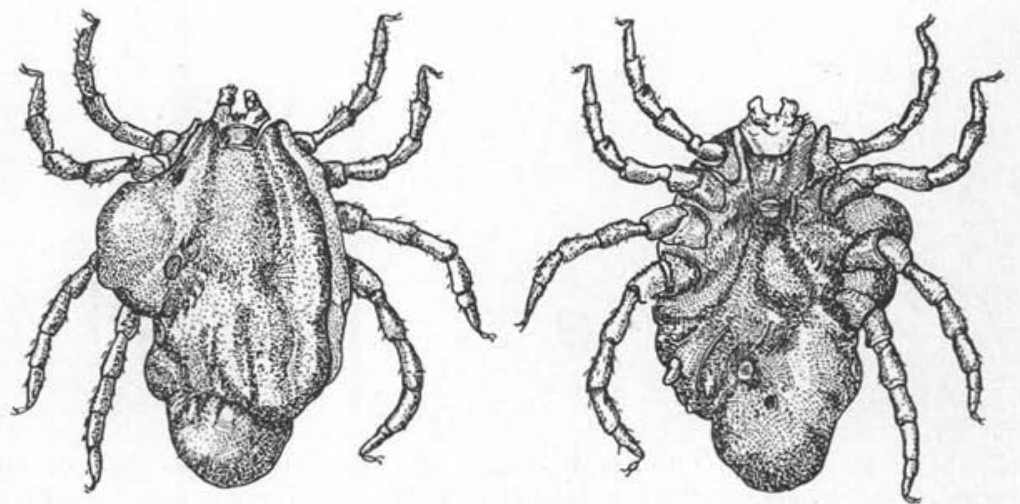

FIG. 15. - Boophilus calcaratus. Mosaïque complète, à prédominance mâle. (D'après E. Pavlovsky).

\section{MOSAIQUES :}

Dans les mosaïques, il n'y a plus de ligne de séparation nette entre les régions mâles et femelles; les plages sont étroitement mélangées sans aucune symétrie et peuvent être extrêmement nom- 
breuses. L'aspect général est donc assez différent de celui des gynandromorphes qui ne possèdent qu'une aire, plus ou moins grande, mais bien délimitée, de chacun des sexes.

\section{Mosaïques complètes :}

Boophilus calcaratus (Birula 1895). Russie. Pavlovsky 1940.

Mosaïque bilatérale, à prédominance mâle (fig. 15).

Uroboophilus cyclops Minning 1934. Guatémala. Schulze 1937.
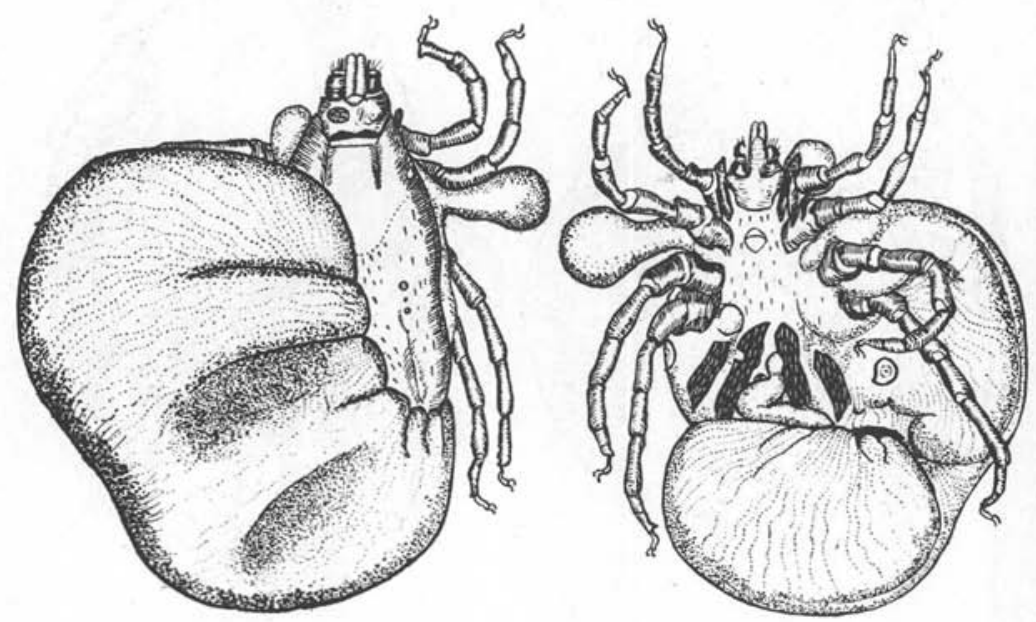

FiG, 16. - Uroboophilus cyclops. Remarquer les expansions latérales de chitine mâle et les enclaves de chitine femelle dans la région anale. (D'après P. Schulze).

Ce curieux exemplaire ressemble un peu au Boophilus de Pavlovsky. Il existe plusieurs expansions de chitine mâle, situées latéralement. Mosaïque à prédominance mâle (fig. 16).

Ixodes dentatus Marx 1899. U.S.A. Smith 1942 (fig. 17).

Haemaphysalis sulcata. Russie. Pavlovsky 1940 (fig. 18).

Très bel exemple de mosaïque bilatérale. Hyalomma savignyi (Gervais 1844). Russie. Pavlovsky et Bernadskaia 1948.

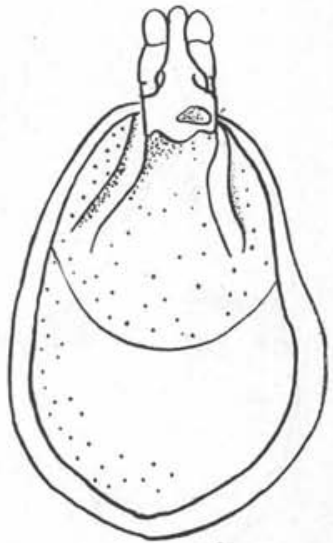

Fig. 17. - Ixodes dentatus. Mosaïque à prédominance femelle. (D'apr. C. Smith). 
Rhipicephalus sanguineus Latr. Brésil. Pereira et Castro 1945 (fig. 19).

L'orifice génital femelle est atrophié ; l'animal n'a pondu qu'un œuf en 37 jours.
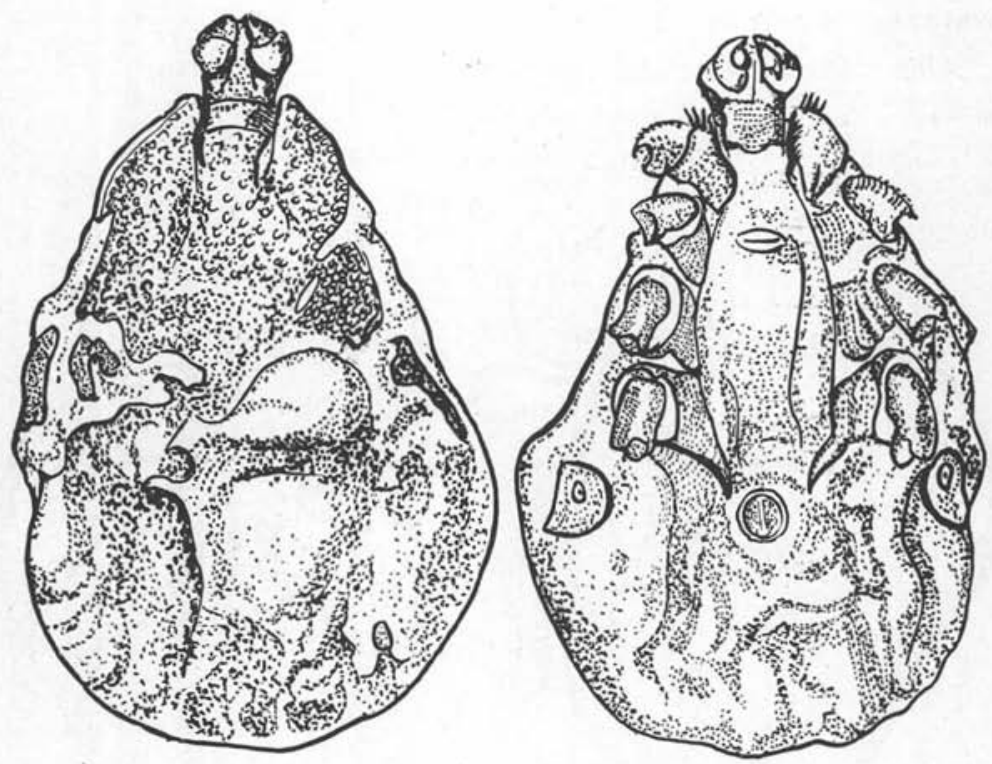

FIG. 18. - Haemaphysalis sulcata. Très bel exemple de mosaïque bilatérale. (D'après E. Pavlovsky).
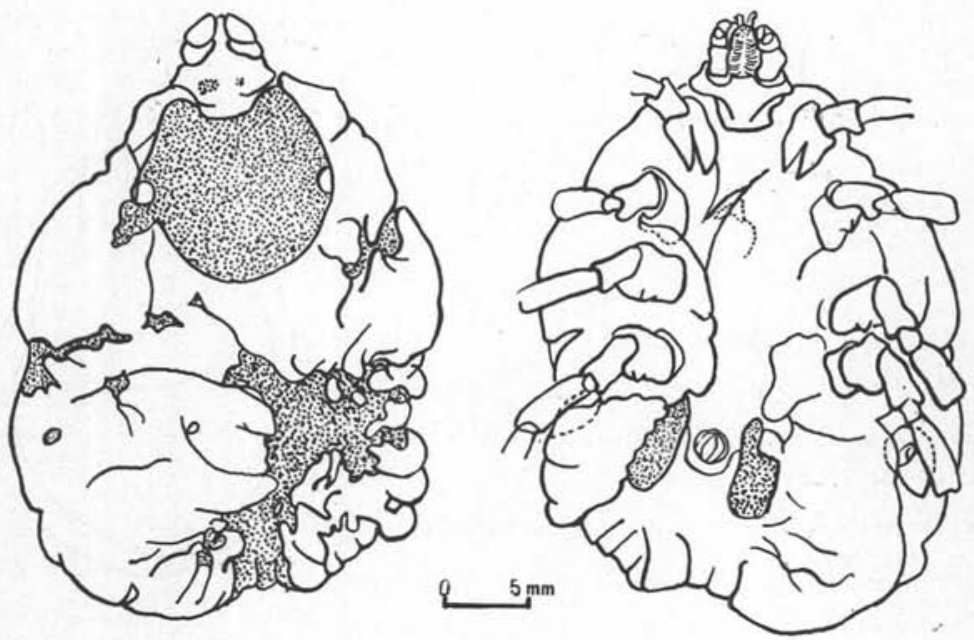

Fıg. 19. - Rhipicephalus sanguineus. Mosaïque bilatérale. (D’après Pereira et Castro). 
Mosaiqques partielles :

1" Dorsales :

Hyalomma asiaticum. Russie. Pavlovsky et Bernadskaia 1948.

Hyalomma marginatum. Russie. Pavlovsky et Bernadskaia 1948.

Hyalomma marginatum Koch. Schulze 1950.

Ilots de chitine femelle en avant de la fovea dorsale droite.

Hyalomma savignyi. Russie. Pavlovsky et Bernadskaia 1948.

Deux exemplaires.

Hyalomma sp. Russie. Pavlovsky et Bernadskaia 1948.

2. Ventrales :

Hyalomma sp. Russie. Pavlovsky et Bernadskaia 1948.

Nous ajouterons à la liste des gynandromorphes un curieux spécimen d'Ixodes ricinus, décrit par Zapletal, en 1957, comme une femelle (fig. 20). L'orifice génital n'est pas figuré, le scutum est de

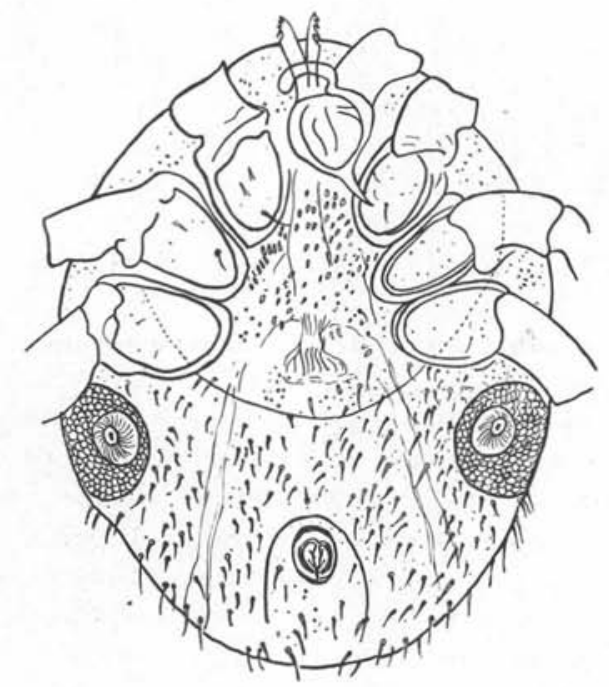

Fit. 20. - Ixodes ricinus. Gynandromorphisme associé à d'autres anomalies. (D'après Zapletal).

type femelle. Le capitulum manque; il y a seulement des chélicères atrophiques, qui paraissent soudés à la première hanche gauche ; celle-ci présente une forte épine de type mâle. A droite, il n'y a que trois pattes, de type femelle. On ne peut classer cet Ixode, qui présente des anomalies trop diverses. 
Nous n'avons pas inclus dans cette liste les gynandromorphes de Pervomaisky, l'auteur ne donnant pas les figures de tous les exemplaires qu'il décrit, et sa classification n'étant pas la même. Nous reproduisons ci-dessous le tableau qu'il donne :

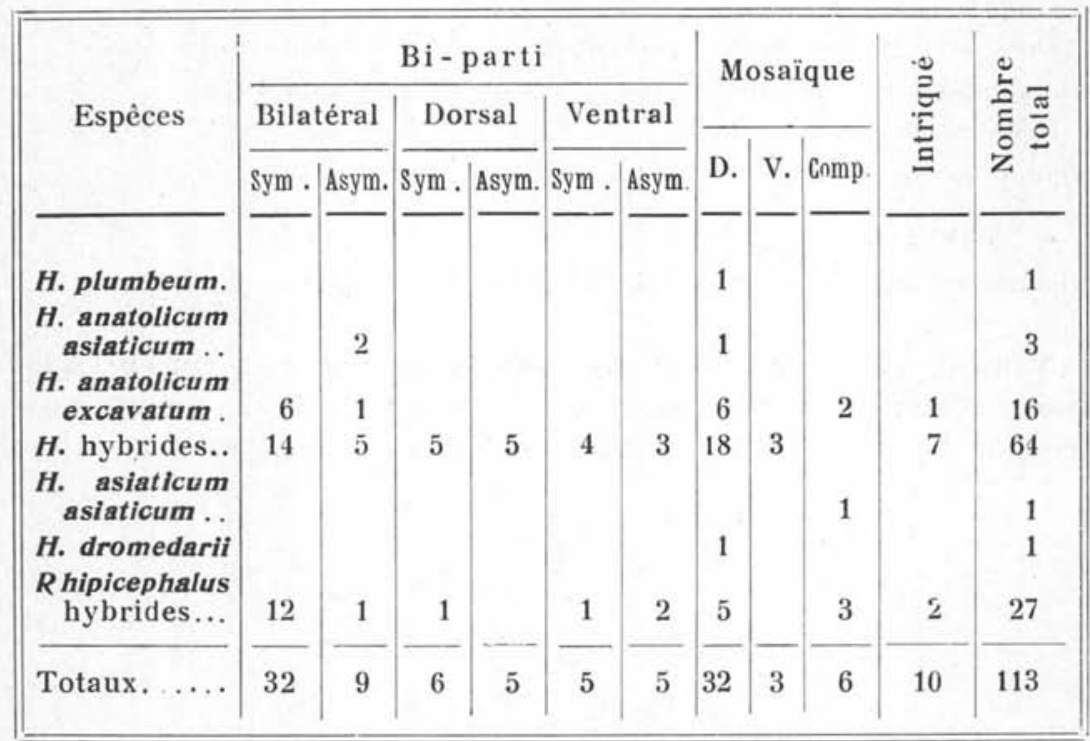

\section{Comportement des gynandromorphes}

Pervomaisky dissèque trois gynandromorphes mi-partis et deux mosaïques. Il en figure deux ; le premier exemplaire, qui a pondu 201 œufs, possède un ovaire assez bien développé ; il y a des œufs de dimensions variables dans les oviductes. Utérus, vagin, spermathèques et glandes accessoires sont normalement développés. Le deuxième exemplaire, disséqué 12 jours après le repas, présente un ovaire plus développé que dans le cas précédent, mais n'a pas d'œufs à maturité dans les voies génitales (fig. 21, A et B).

Les autres spécimens sont disséqués à jeun, aussi ont-ils des organes génitaux (femelles) de dimensions réduites, mais normaux.

Il est à remarquer que, lors de toutes les dissections effectuées, ce sont toujours des organes femelles que l'on trouve, même quand il s'agit d'animaux n'ayant pas extérieurement de prédominance femelle. 

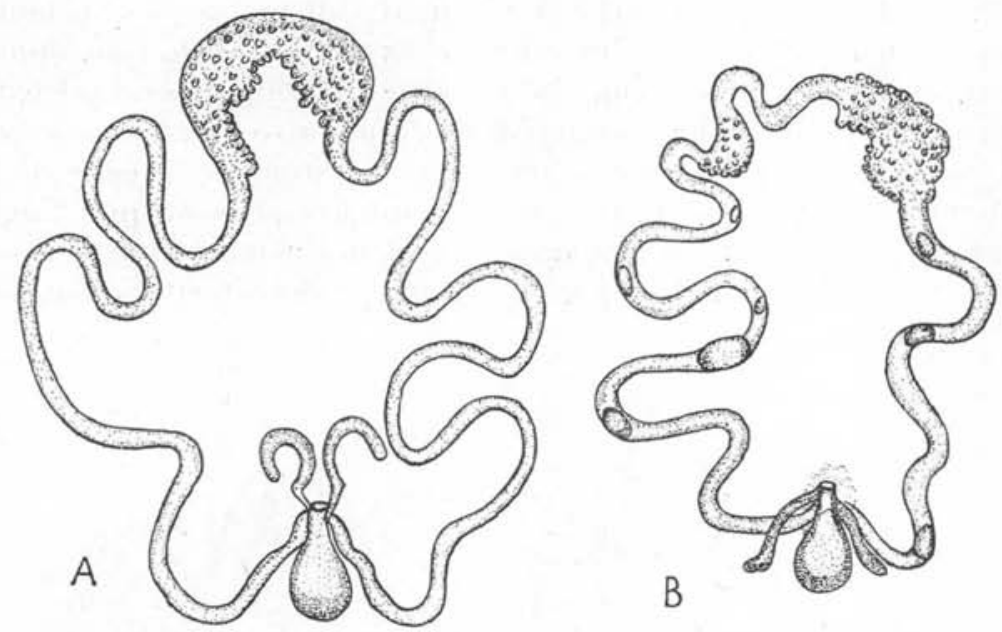

Fıg. 21. - A et B, Dissection de deux appareils génitaux femelles provenant de gynandromorphes. (D’après Pervomaisky).

\section{INTERSEXUALITÉ}

Avant de discuter l'origine du gynandromorphisme, nous terminerons la liste des anomalies sexuelles en signalant quelques cas d'intersexualité. Rappelons qu'il s'agit ici d'un phënomène tout différent de celui que nous avons étudié précédemment : il survient tardivement dans la vie de l'individu et il est d'origine hormonale.

Hyalomma savignyi (Gervais 1844), Palestine, Feldman-Muhsam. - Exemplaire mâle ayant subi une influence féminisante locale : le stigmate situé dans cette région est devenu femelle, tandis que les écussons et les festons situés du même côté ont subi une réduction de taille importante (fig. 22).

C'est le premier cas indiscutable que nous connaissions, mais il est possible que des cas semblables aient passé inaperçus : il faudrait pouvoir passer en revue tous les exemplaires présentant une asymétrie des écussons; une réduction de taille, sans autre anomalie des caractères sexuels secondaires, devra orienter les recherches dans ce sens. Il serait intéressant de disséquer de tels individus, pour avoir des renseignements sur l'état de l'appareil génital.

Le mème auteur nous signale un deuxième cas, toujours chez H. savignyi : les caractères femelles n'apparaissent ici que dans la partie antérieure du scutum. 
Chabaud et Choquet (1952), en étudiant l'allométrie des variants sexuels chez les Ixodidæx, obtiennent, chez des mâles de Hyalomma excavatum hyponutris (nymphes arrachées de leur hôte avant leur gorgement complet), une réduction des écussons et des stigmates ; un exemplaire présente même d'un côté un stigmate femelle lié à l'absence des écussons de ce côté. Ces auteurs pensent qu'il s'agit ici d'un phénomène métabolique et non hormonal. Malheureusement, ils n'ont jamais pu par la suite reproduire cette anomalie.

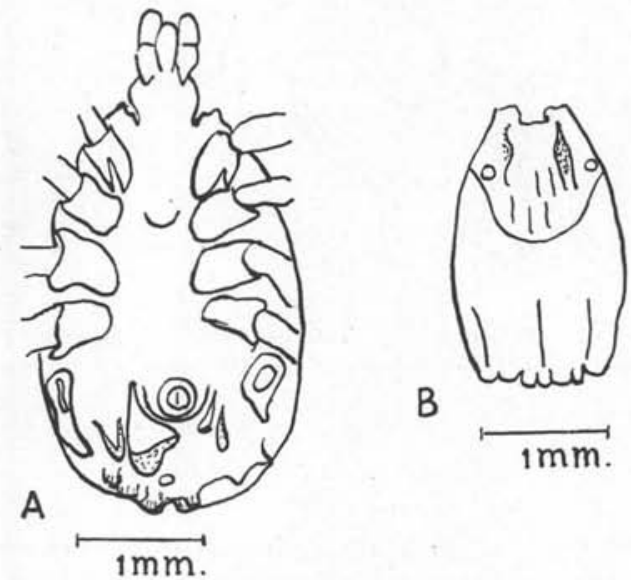

Fig. 22. - Hyalomma savignyi. Deux intersexués. (D’après Feldman-Muhsam).

Pervomaisky trouve également un certain nombre de mâles à écussons réduits dans ses populations d'hybrides. Il en conclut que le gynandromorphisme est la limite extrême de la variabilité observée chez ses hybrides. Il est difficile de savoir à quoi attribuer cette variabilité. Il est possible que ce soit un phénomène d'intersexualité pure, la production hormonale étant troublée chez ces animaux de constitution particulière.

\section{Théories du gynandromorphisme applicables oux Ixodidés}

Le gynandromorphisme, connu et étudié depuis longtemps chez les Insectes principalement, a été l'objet de bien des théories plus ou moins contradictoires. On sait maintenant que cette monstruosité provient d'une répartition anormale des chromosomes sexuels qui peut se produire dans l'œuf, dès les premiers stades du développement, ou bien à un stade ultérieur ; dans le prèmier cas, on aura des gynandromorphes, dans le second cas, des mosaïques. 


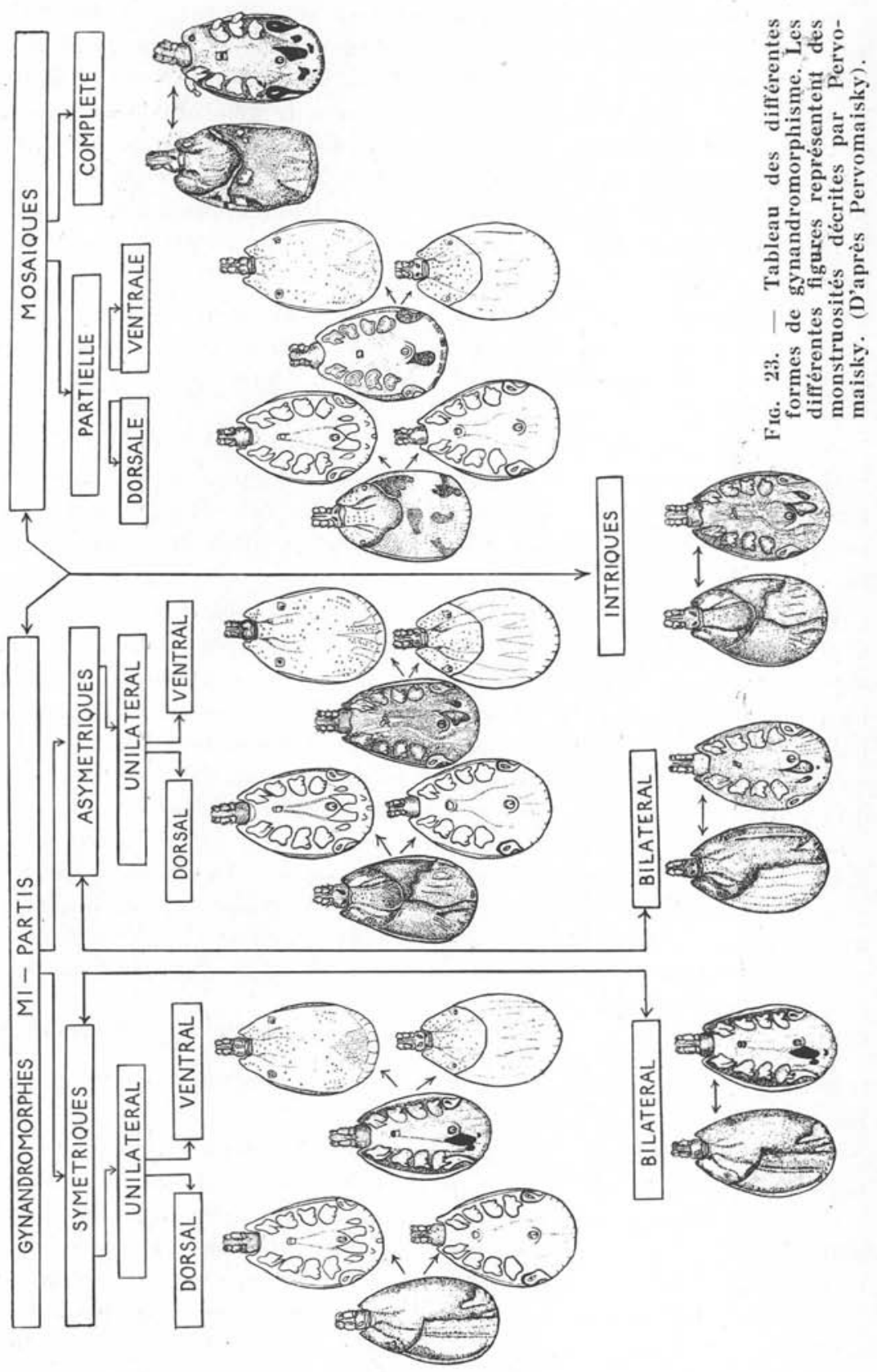


Sur le processus même présidant à la répartition chromosomique, il reste encore un certain nombre de théories en présence, s'appuyant chacune sur des faits précis, et il semble bien, comme le dit Vandel (1931), que « des phénomènes de gynandromorphisme qui sont des processus tératologiques, done particuliers, doivent, dans chaque cas, recevoir une explication particulière. Il ne saurait $\mathrm{y}$ avoir de théorie générale du gynandromorphisme, mais seulement des explications partielles s'appliquant à des cas particuliers $\gg$.

La plupart des auteurs ayant décrit des gynandromorphes chez les Tiques ont essayé de déterminer la cause de cette anomalie, sans toutefois beaucoup approfondir cette question qui dépassait le cadre de leurs recherches.

Nous citerons d'abord l'opinion de Teresa Joan (1916), à qui revient la description du premier gynandromorphe connu chez les Ixodidés ; son hypothèse n'a d'ailleurs qu'un intérêt purement historique, car elle méconnaît totalement la question des chromosomes sexuels.

Pour T. Joan, le sexe serait fonction des facteurs externes agissant au moment de la fusion de l'ovule et du spermatozoïde. Elle suppose que chez son Amblyomma, qui est un gynandromorphe mi-parti, chaque moitié du corps dérive d'un des deux premiers blastomères D et G, D donnant la moitié droite du corps et G la moitié gauche; les échanges nutritifs de D ayant été plus intenses que ceux de G, D a évolué vers le sexe femelle qui possède généralement une plus grande abondance de réserves, et $\mathrm{G}$ vers le sexe mâle. Le facteur interne, susceptible de faire varier l'intensité des échanges, serait la quantité plus ou moins grande de chromatine du noyau. Chez certains Insectes, les œufs qui ont été fécondés par des spermatozoïdes à chromosome accessoire donnent des femelles, car le rapport $\frac{\text { chromatine }}{\text { cytoplasme }}$ s'est trouvé augmenté. Nous nous trouvons évidemment fort loin des théories modernes sur la sexualité.

Schulze (1933), après avoir rappelé les différentes théories données par Goldschmidt, estime qu'en l'absence de données précises sur la constitution chromosomique des Tiques, il est difficile de les rattacher à un type donné. Toutefois, en s'appuyant sur les phénomènes de parthénogénèse connus chez Amblyomma rotundatum, il propose la théorie suivante : l'une des cellules de l'œuf peut être haploïde et l'autre, une fois fécondée, diploïde, ce qui nous ramène au type Abraxas de Concaster. En réalité, contrairement à ce que 
pensait Schulze, la parthénogénèse parait être absolument constante chez $A$. rotundatum : le mâle n'a jamais été signalé et les nombreux élevages de cette espèce, faits au laboratoire de parasitologie de la Faculté de Médecine de Paris, ont toujours donné uniquement des femelles; on n'a naturellement pas trouvé de gynandromorphes chez ces animaux. L'hypothèse de Schulze n'est donc nullement confirmée pour $A$. rotundatum et parait, a fortiori, inapplicable pour l'ensemble des Ixodinés.

En 1935, Oppermann noús montre que, chez Argas columbarum, le mâle est hétéro-gamétique. Il en est probablement de même chez les Ixodinx. Schulze (1937) tient compte de cette nouvelle acquisition, et assimile les Tiques, soit au type Bombyx mori de Goldschmidt et Katsuki, soit au type Habrobracon de Whiting.

Pereira et Castro (1945) rejettent l'hypothèse de Schulze, comme étant peu probable, et adoptent celle de l'école de Morgan qui leur paraît s'adapter complètement aux Ixodes. Selon eux, la perte précoce d'un chromosome $\mathrm{X}$ donnerait naissance à un gynandromorphe mi-parti, l'élimination plus tardive du chromosome donnerait une mosaïque à prédominance femelle. La répétition de ce phénomène chez le même individu donnerait une mosaïque typique comme chez l'Uroboophilus de Schulze et le Rhipicephalus de Pereira.

Les Tiques sont génétiquement trop mal connues pour que nous puissions avec certitude les assimiler à tel ou tel type. Nous ne pouvons que procéder par comparaison avec des animaux extrêmement différents.

Nous savons que, chez les Argas, le mâle est hétéro-gamétique ; il n'est pas sûr qu'il en soit ainsi chez tous les Ixodes. Nous avons consulté plusieurs ouvrages sur la spermiogénèse, mais aucun ne nous donna de renseignements précis. Nordenskiöld (1920) indique qu'il doit y avoir 14 chromosomes chez Ixodes ricinus, ces chromosomes étant en forme de bâtonnets légèrement incurvés. Mlle Tuzet et Millot (1937) donnent le nombre de 12 chromosomes pour Hyalomma aegyptium et Rhipicephalus bursa ; chez la première de ces Tiques, il existe un grand chromosome sexuel allongé; chez la seconde, on ne retrouve pas cette formation, il y a toutefois un bâtonnet plus allongé que les autres.

Nous voyons que le nombre et la forme des chromosomes changent d'un genre à l'autre. Nous supposons toutefois que l'hétérogamétie mâle ou femelle est un caractère plus constant, puisqu'on retrouve le même type dans des groupes assez étendus.

La théorie de la duplicité de la cellule initiale ne nous parait pas satisfaisante. Nous pensons comme Vandel que si le gynandromor- 
phe n'est que le cas limite d'un monstre double, les formations surnuméraires devraient se rencontrer fréquemment; or, les seuls signes de duplication que nous ayons vus sont la petite patte supplémentaire greffée sur la deuxième patte droite de l'Amblyomma dissimile de E. Brumpt, et la patte schizomèle d'un des Hyalomma gynandromorphes de Pervomaisky. Ces animaux ne ressemblent nullement aux monstres doubles que nous verrons plus tard; ceuxci se présentent sous un aspect cordiforme, et le premier signe de duplication observée est l'apparition de deux anus.

De plus, on ne peut guère admettre dans le cas présent une régulation faisant disparaitre toutes traces de duplication entre la larve et l'adulte, comme chez les phasmides de Cappe de Baillon : en effet, chez tous les monstres doubles d'Ixodes dont on a pu suivre l'évolution, on a constaté que l'anomalie persistait pendant toute la vie de l'animal, sans aucune régression.

Les différentes théories s'appuyant sur l'évolution d'une cellule binucléée peuvent s'appliquer dans les cas de gynandromorphes mipartis, mais n'expliquent pas les mosaïques.

La parthénogénèse est un fait beaucoup trop accidentel chez les Ixodes pour que l'on puisse admettre le développement d'une partie de l'individu par ce processus.

L'hypothèse de l'élimination d'un chromosome $\mathrm{X}$ nous parait ici la plus séduisante. Si cette élimination se produit au cours de la première mitose de segmentation, il se formera un protogynandre ; au cours de la deuxième, un deutérogynandre, etc...; si elle a lieu à un stade plus tardif, on aura une mosaïque à prédominance femelle, comme c'est le cas le plus fréquent. Nous avons toutefois une prédominance mâle chez le Boophilus calcaratus de Pavlovsky. On peut supposer dans ce cas que l'élimination d'un chromosome X, qui s'est produite au premier stade, a pu se produire à nouveau à un stade ultérieur, provoquant l'apparition de nouvelles aires mâles dans la région femelle. Si l'élimination du chromosome est due à un gène analogue au gène minute-n de la drosophile, rien n'empêche d'admettre que le phénomène se reproduise à différentes reprises.

Nous pouvons tenir compte encore de l'idée de Vandel : l'action du porte-greffe sur son greffon, surtout lorsqu'elle s'exerce dès le début du développement, peut probablement expliquer les aspects intersexués que l'on rencontre chez certains gynandromorphes. C'est sans doute un phénomène de cet ordre qui pourrait rendre compte de la mosaïque d'éléments mâles et femelles que nous avons vue chez l'Amblyomma scutatum de Schulze. 
S'il en était besoin, la théorie génétique serait renforcée par les expériences de Pervomaisky : en effet, il apparait comme tout à fait concluant de rencontrer un nombre particulièrement élevé de gynandromorphes (91 en deux expériences) chez des hybrides dont la constitution chromosomique est forcément instable.

Il est généralement très difficile d'obtenir des croisements fertiles chez les Ixodidæ. Pervomaisky a sans doute eu la chance de croiser des espèces de constitution chromosomique suffisamment proche.

La portée et la signification réelle de ses expériences n'en sont pas moins grandes.

\section{Monstruosités doubles}

Les monstres doubles sont assez largement représentés chez les Ixodoidea : nous connaissons une cinquantaine de cas, le plus souvent isolés. Cependant, Aboim-Inglez, en 1942, a trouvé dans un élevage d'Haemaphysalis punctata, provenant de la ponte d'une seule femelle (1.764 larves), 26 exemplaires présentant des duplications plus ou moins marquées. Ce fait unique a permis à l'auteur de classer ces monstruosités parmi cinq types selon le degré de la duplication :
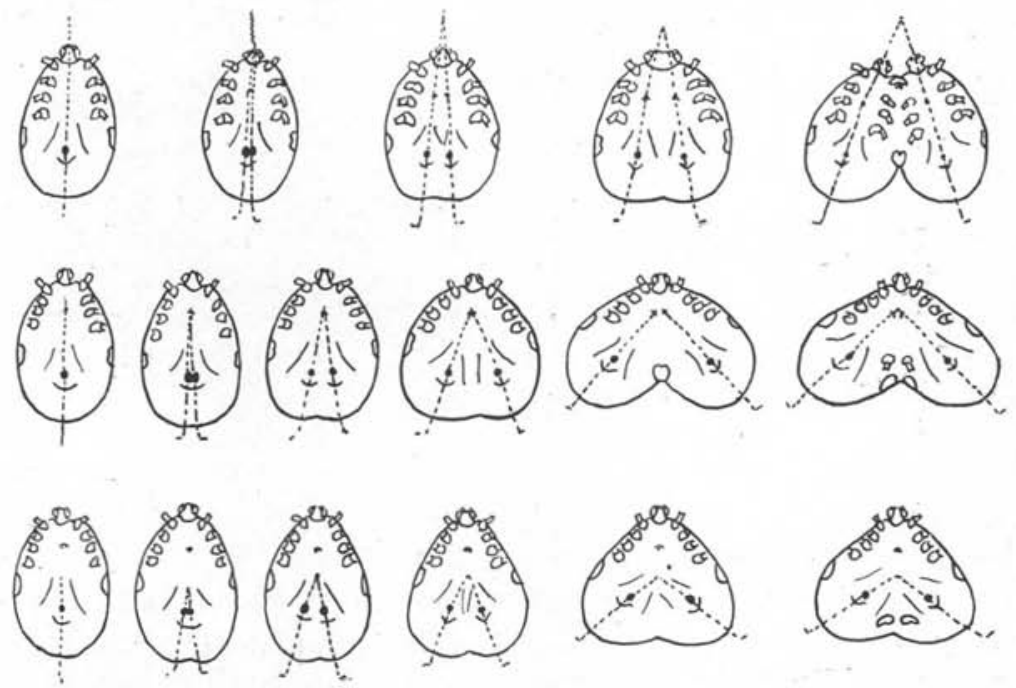

Fig. 24. - Tableau théorique de la formation des monstres doubles. (D’après Aboim-Inglez). 
$1^{\circ}$ Type à 2 anus, 4 stigmates, 2 orifices génitaux, 2 pattes supplémentaires.

2 . Type à 2 anus, 4 stigmates, 2 orifices génitaux.

$3^{\circ}$ Type à 2 anus, 3 stigmates.
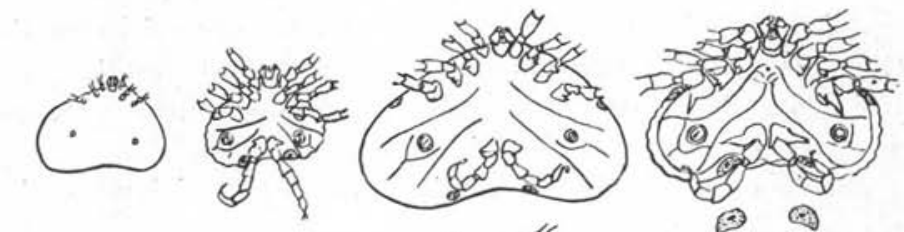

10
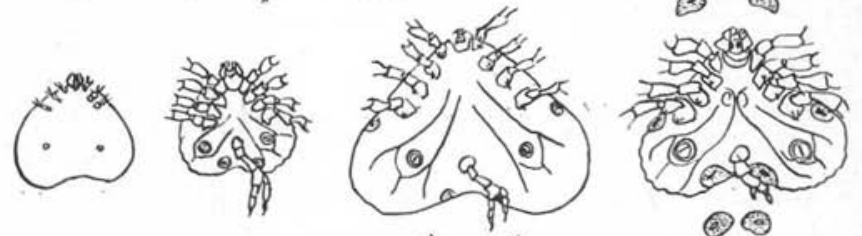

19
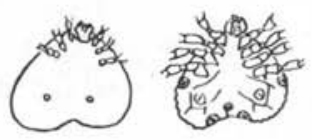

(1)

(2) 6
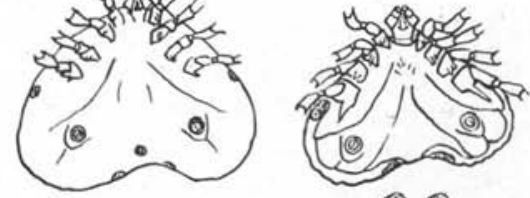

(2) (2)
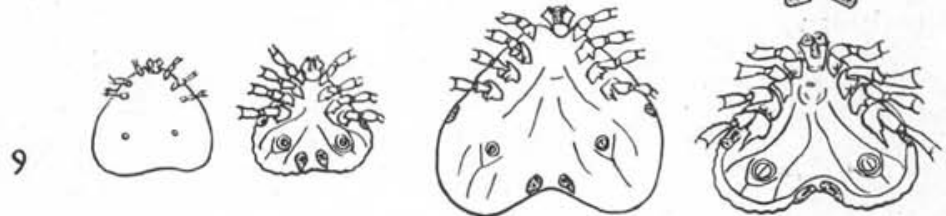

ब(2)
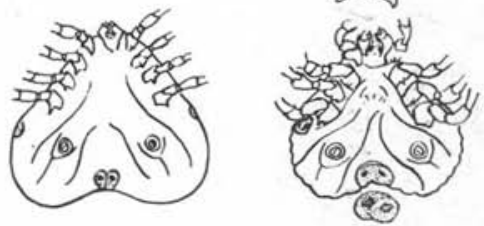

FIG. 25. - Haemaphysalis punctata. Cinq exemplaires présentant des caractères de duplication très marquée. (D'après Aboim-Inglez).

$4^{\circ}$ Type à 2 anus.

$5^{\circ}$ Type à bord postérieur élargi ou échancré.

Aboim-Inglez a pu élever plusieurs exemplaires jusqu'à l'état adulte, suivant ainsi l'évolution de la monstruosité ; il constate que celle-ci ne régresse que très rarement, il y a au contraire acquisi- 
tion de stigmates et de pattes surnuméraires au stade nymphal (en même temps qu'apparaissent les stigmates normaux et la quatrième paire de pattes). Chez l'adulte, apparait parfois un deuxième orifice génital.
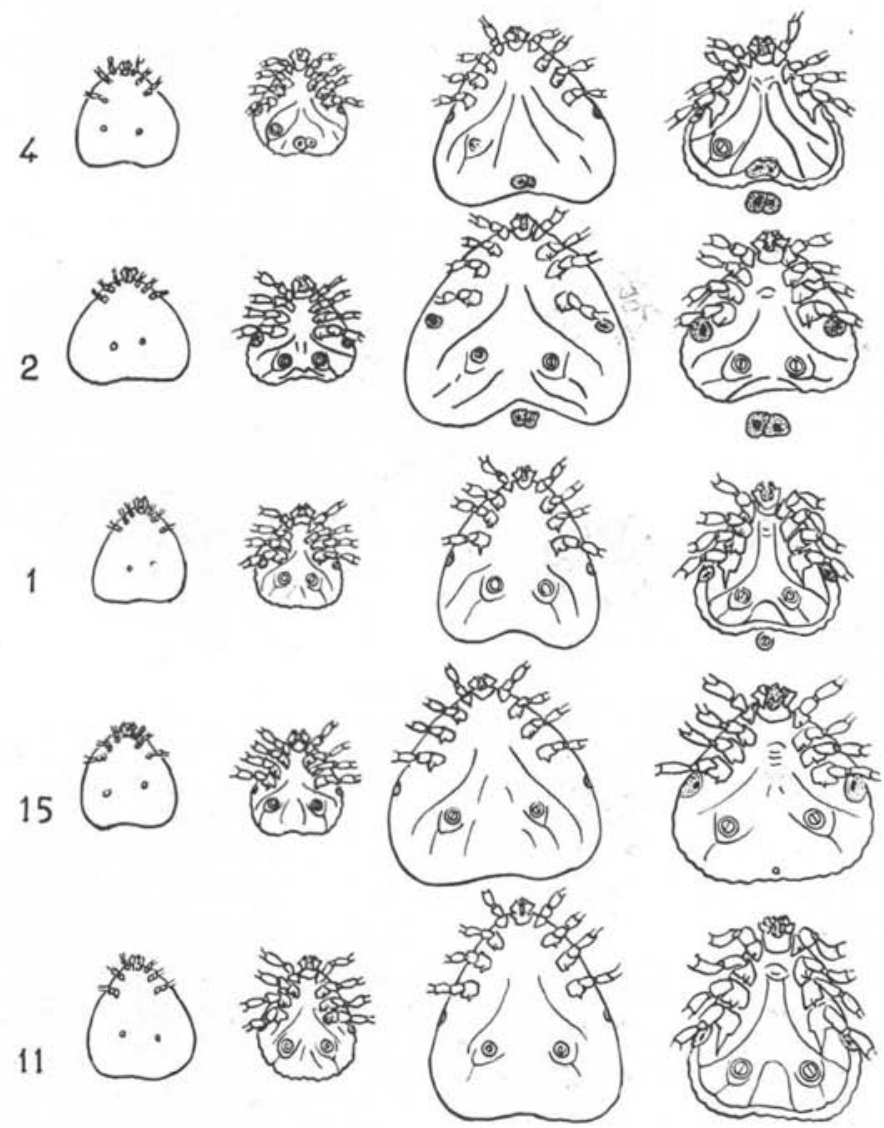

Fig. 26. - Haemaphysalis punctata. Cinq autres exemplaires avec des degrés de duplication déeroissants. (D'après Aboim-Inglez).

L'auteur ayant étudié toutes les variétés de duplication possibles, sauf celle de la tête, pense que l'anomalie débute postérieurement et progresse d'arrière en avant. Il imagine que les plans de symétrie de chacune des moitiés de l'animal dédoublé forment un dièdre d'ouverture variable selon l'importance de la duplication. Le point de rencontre des deux axes est situé à des niveaux 
variables : plus il est intérieur, plus la disjonction est importante. On peut ramener la formation hypothétique de ces monstres à trois types :

16
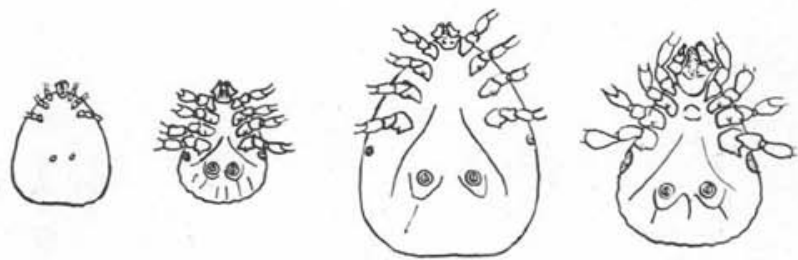

18
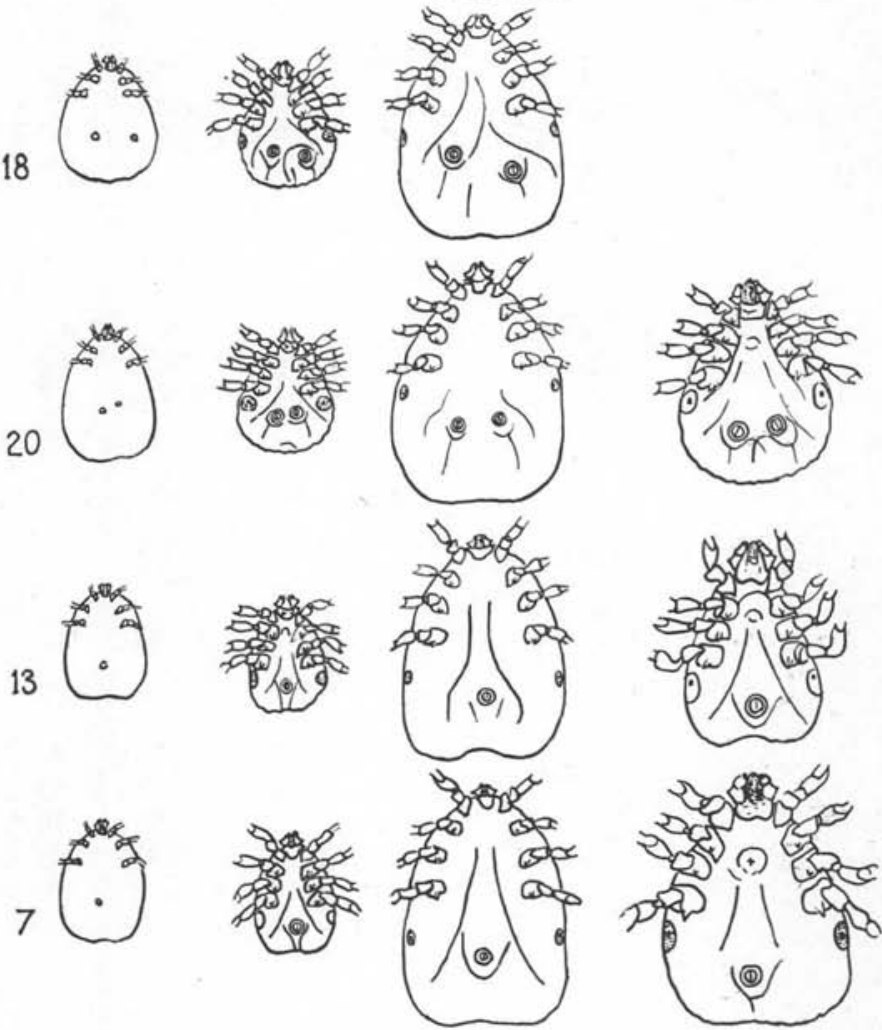

Fig. 27. - Haemaphysalis punctata. Exemplaire à deux anus ou simplement cordiformes. (D'après Aboim-Inglez).

$1^{\circ}$ point de rencontre des deux axes en avant du corps : devrait donner des monstres à deux têtes (jamais rencontrés);

$2^{\circ}$ point de rencontre des deux axes entre le capitulum et l'orifice génital (monstres à deux orifices génitaux, très rares); 
$3^{\circ}$ point de rencontre des deux axes entre l'orifice génital et l'anus (le plus fréquent) (fig. 24).

Nous allons donner maintenant la liste des monstres doubles connus ou inédits en les classant dans six groupes selon leur degré de duplication :

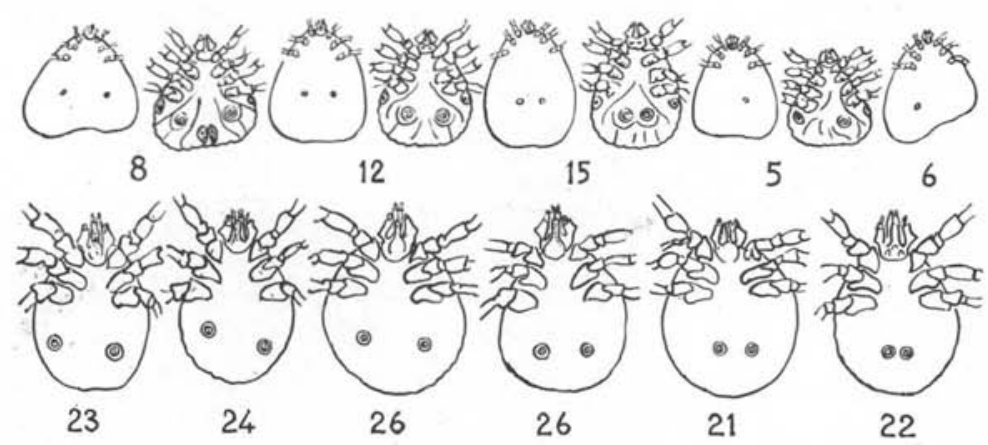

Fig. 28. - Haemaphysalis punctata. Larves et nymphes. Deux larves ont un seul anus, mais asymétrique.

Type I. - Un seul anus, médian ou non, partie postérieure du corps élargie ou cordiforme.

Nous faisons figurer dans ce groupe des exemplaires qui n'avaient pas été décrits comme monstres doubles par leurs auteurs, mais qui trouvent place indiscutablement à côté des spécimens d'Aboim-Inglez.

Amblyomma rotundatum. Cinq femelles décrites par Beaurepaire-Aragao en 1912 (fig. 29).

Boophilus decoloratus. Mâle décrit par Santos Diaz en 1947 (fig. 30).

Haemaphysalis punctata (Canestrini et Fanzago 1877). Deux femelles, une nymphe et une larve, décrites par Aboim-Inglez en 1942.

Ces deux dernières ont un anus symétrique (fig. $27, \mathrm{n}^{\circ} 7,13$; fig. 28 , $\mathrm{n}^{\circ}$ 5-6).

Ixodes ricinus. Tchécoslovaquie. Cerny 1957.

Larve cordiforme (fig. 30 bis).

Rhipicephalus sanguineus Latreille 1904.

Larve provenant d'un de nos élevages.

Un exemplaire cordiforme qui est malheureusement mort avant de muer (fig. 31).

Argas persicus Oken 1818. Femelle cordiforme décrite par Pavlovsky en 1940 (fig. 32).

Ann. de Parasitologie, $\mathrm{T}$. XXXIV, $\mathrm{N}^{\circ} 1-2 .-1959$. 

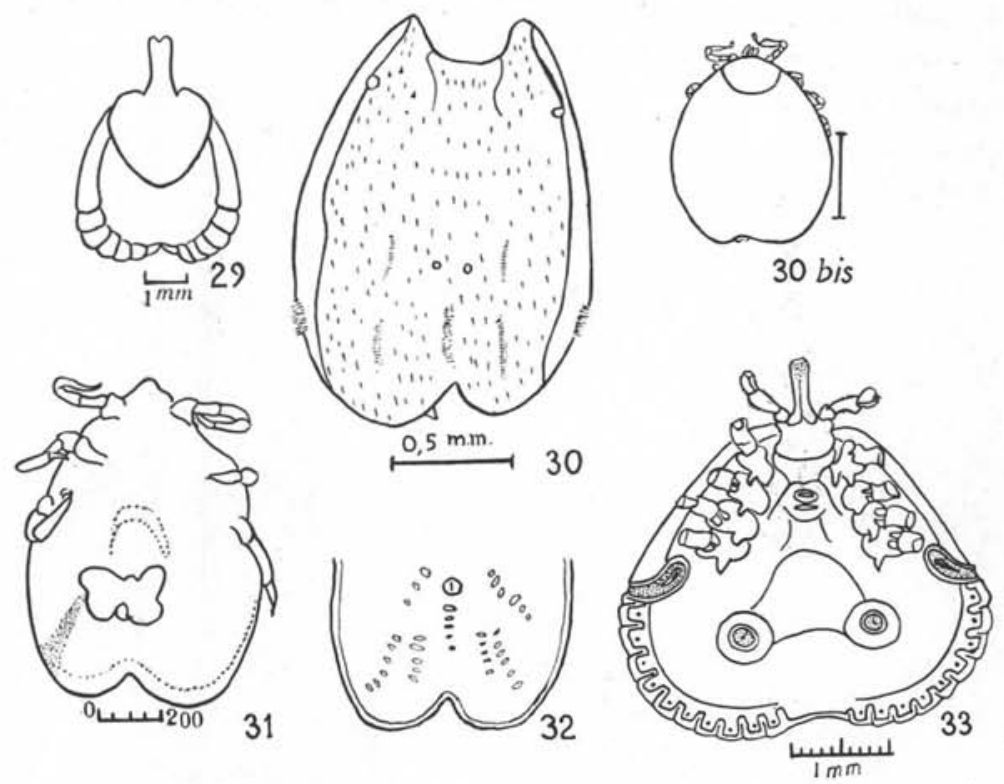

FIG. 29. - Amblyomma rotundatum. Femelle cordiforme. (D'après Beaurepaire-Aragao) ; FIG. 30. - Palpoboophilus decoloratus. Mâle cordiforme. (D'après T. Santos Dias) ; FIG. 30 bis. - Ixodes ricinus. Larve cordiforme. (D’après Cerny) ; Fig. 31. - Rhipicephalus sanguineus. Larve cordiforme, anus asymétrique. (Original) ; FIG. 32. - Argas persicus. Femelle cordiforme. (D'après Pavlovsky); FIG. 33. - Amblyomma dissimile. Mâle à deux anus et double série de festons. (D'après E. Brumpt).

\section{Type II. - Deux anus.}

C'est le groupe le plus nombreux; il comporte un certain nombre de larves, qui auraient peut-être montré des duplications plus importantes au cours de leur développement. On peut admettre que, plus les anus sont éloignés l'un de l'autre, plus le spécimen aura de chances d'accéder aux types plus complexes.

Amblyomma dissimile Koch 1844. Nymphe décrite par E. Brumpt en 1934.

Elle donne ensuite un mâle du même type (fig. 33).

Boophilus decoloratus Koch 1844. Mâle d'un élevage du Cap, décrit par Warburton et Nuttall en 1909.

Les écussons anaux sont dédoublés, mais pas les adanaux (fig. 34).

Boophilus calcaratus (Birula 1895). Femelle décrite par Pavlovsky en 1940 (fig. 34 bis).

Hyalomma sp. (1). Femelle venant d'Afrique du Nord, étudiée par Sénevet en 1922 (fig. 35).

(1) Nous désignons comme Hyalomma $s p$. toutes les Tiques de Mammifères décrites sous le nom de Hyalomma aegyptium, cette dénomination étant maintenant réservée aux seules Tiques de Tortues. 
Hyalomma anatolicum Koch. Russie. Trois exemplaires décrits par Pervomaisky.

Femelle à deux anus.

Mâle à deux anus avec un double système d'écussons complet.

Mâle à deux anus, dont le dédoublement des écussons est moins complet que précédemment : deux écussons adanaux, pas de subanaux,
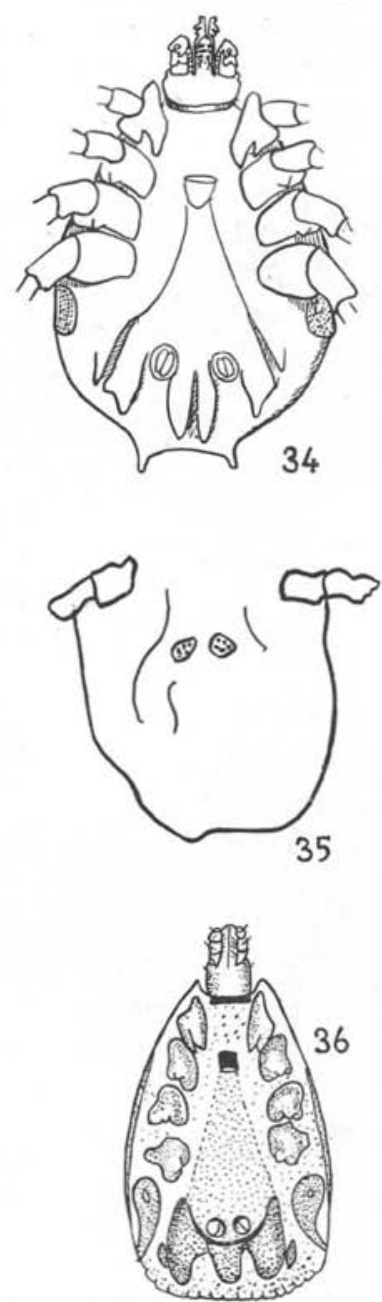
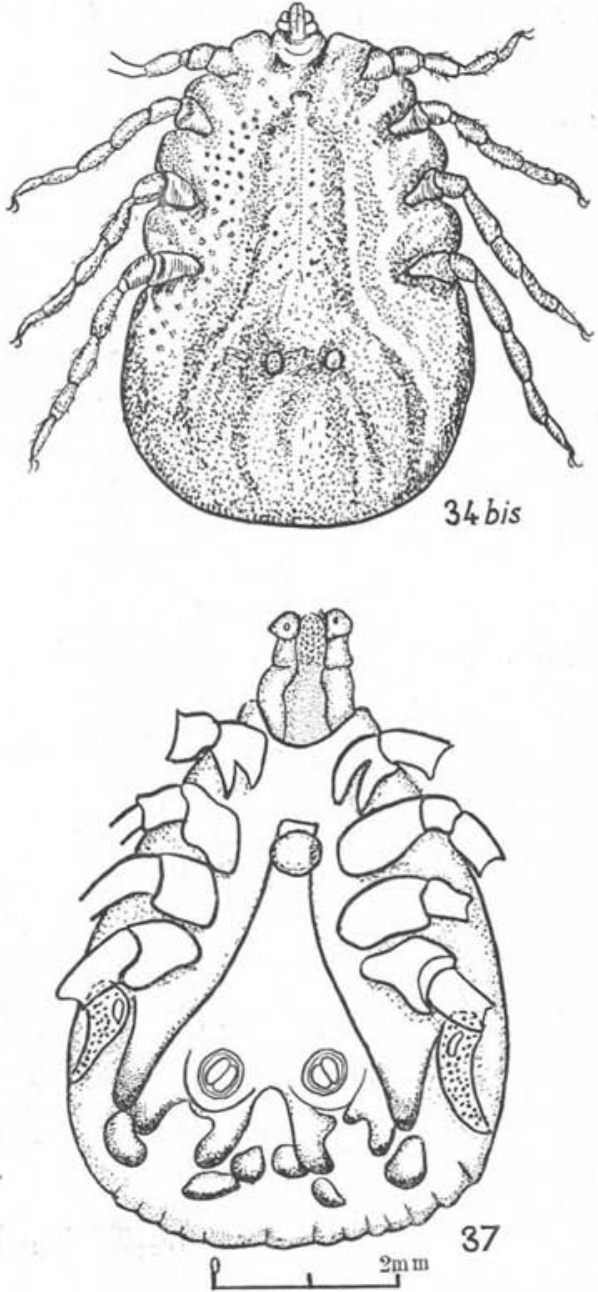

Fig. 34. - Boophilus decoloratus. Dédoublement de l'anus, des écussons et des épines caudales. (D'après Warburton et Nuttall); FIG. 34 bis. - Boophilus calcaratus. Femelle à deux anus rapprochés. (D’après Pavlovsky) ; Fig. 35. - Hyalomma sp. Femelle à deux anus assez rapprochés. (D’après Sénevet) ; Fig. 36. - Hyalomma anatolicum. Dédoublement incomplet des écussons : trois écussons anaux reliés par leur base. (D'après Pervomaisky); Fig. 37. - Hyalomma dromedarii. Deux systèmes d'écussons au complet. (Original). 
trois écussons anaux reliés par leur base, dont un médian au-dessous de la région anale (fig. 36 ).

Hyalomma dromedarii (Koch 1844).

Mâle provenant de la collection du Laboratoire de Parasitologie, non décrit. Il existe deux systèmes complets d'écussons, normalement développés (fig. 37).

Haemaphysalis punctata. Portugal. Décrits par Aboim-Inglez en 1942.

Un mâle, deux femelles, trois nymphes et six larves. L'une des larves porte un fragment de patte supplémentaire sur le trochanter de la première patte droite (fig. 25-28).
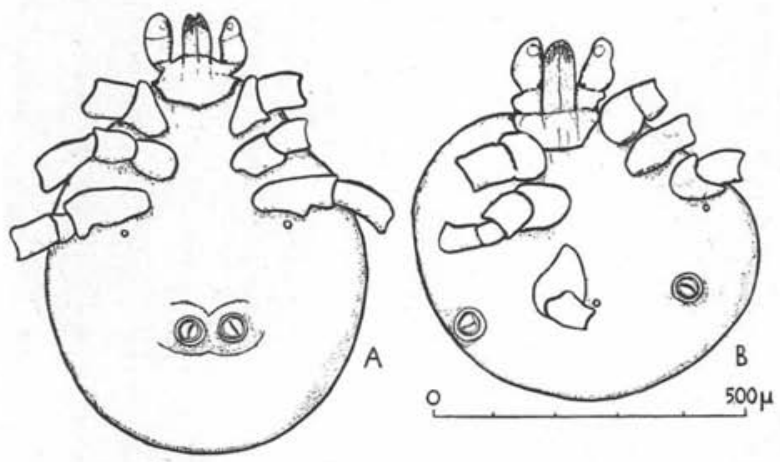

FIG. 38. - Haemaphysalis punctata. A et $\mathrm{B}$, deux larves à deux anus. (Original).

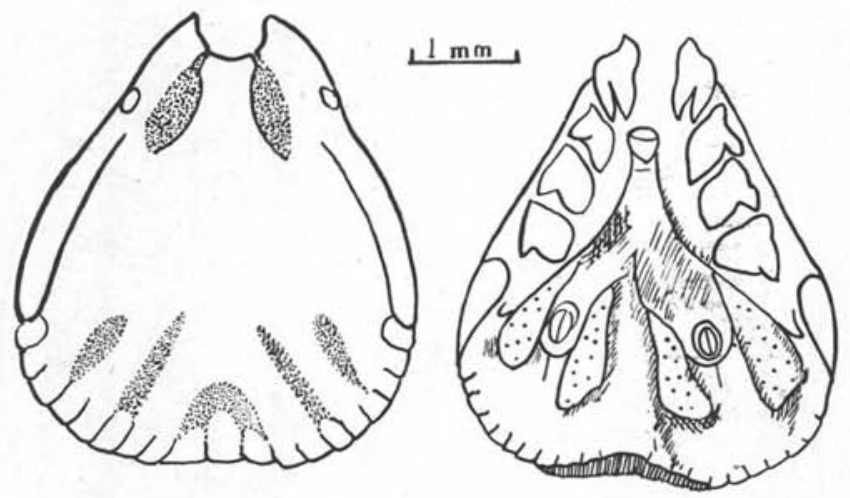

Fig. 39. - Rhipicephalus appendiculatus. Dédoublement des écussons anaux. (D’après Nuttall).

\section{Haemaphysalis punctata. Portugal.}

Deux larves récoltées dans le même élevage que précédemment, non décrites (fig. $38, \mathrm{~A}$ et $\mathrm{B}$ ).

L'une a ses deux anus proches de la ligne médiane, l'autre les a beaucoup plus écartés, et le corps est très élargi postérieurement, ce qui permet de penser que l'adulte aurait été du type IV ou V. 
Rhipicephalus appendiculatus Neumann 1908. Le Cap. Mâle décrit par Nuttall en 1914.

Seuls les écussons anaux sont dédoublés (fig. 39).

Ornithodorus moubata (Murray 1877). Deux nymphes d'un élevage signalées par G. Robinson en 1943.

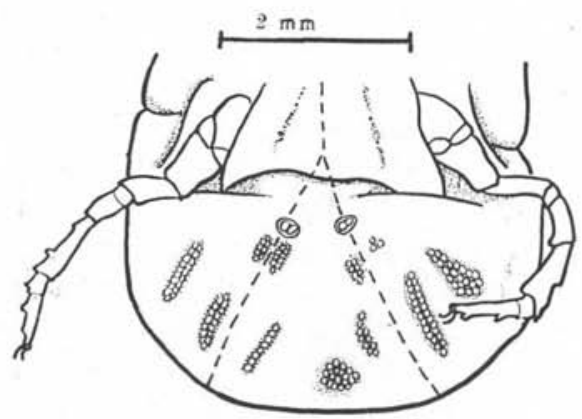

Fig. 40. - Ornithodorus moubata. Femelle à deux anus. Dédoublement des disques anaux. (D'après Robinson).

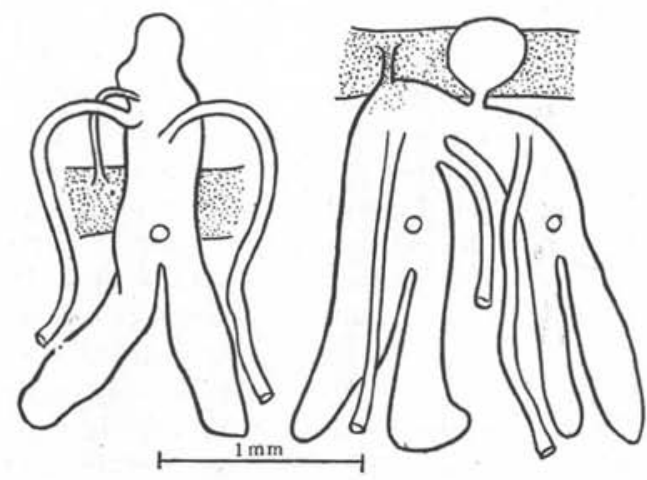

F1G. 41. - Ornithodorus moubata. Dissection : à gauche, sac rectal normal : à droite, sac rectal dédoublé de l'exemplaire précédent. (D'après G. Robinson).

Une nymple au cinquième stade a donné une femelle et l'autre, au troisième stade, un mâle. L'aspect des deux individus est à peu près le même, et seule la femelle a été représentée (fig. 40). Il y a deux anus et deux paires de disques anaux. Toutes les plaques correspondant à l'insertion des muscles sur la cuticule sont dédoublées, sauf une au centre. L'orifice génital est simple, l'accouplement a eu lieu normalement et a été fertile.

L'auteur a étudié l'anatomie interne de ces animaux et a pu faire plusieurs constatations intéressantes : 
- Chez la femelle, au lieu d'un ovaire médian continué par deux oviductes, il trouve deux ovaires latéraux munis chacun d'un seul oviducte. Le sac rectal est divisé seulement partiellement, le sac droit est normal, avec deux tubes de Malpighi, le sac gauche ne possède qu'un tube et n'est pas relié à l'intestin; à la place du rectum se trouve une petite vésicule de la grosseur d'un œuf mûr environ. Les deux anus sont fonctionnels ; le rectum est un tube creux, ce qui est tout à fait inhabituel, et les fèces sont rougeâtres au lieu d'avoir la couleur crème du contenu malpighien.

- Chez le mâle, le testicule est aussi divisé en deux; le sac rectal est divisé et présente trois tubes de Malpighi et deux ouvertures anales fonctionnelles. Le rectum est normal (fig. 41).

Hyalomma mauritanicum Sénevet 1932. Algérie. Nymphe décrite par

Sergent et Poncet en 1941.

Cette nymphe possède deux anus. Les auteurs indiquent que les deux stigmates postérieurs sont restés distincts; la photo donnée ne les montre pas. Après la mue, le mâle présente deux anus, mais les écussons ne sont pas dédoublés, et il semble bien qu'il n'y ait pas de stigmates sur-

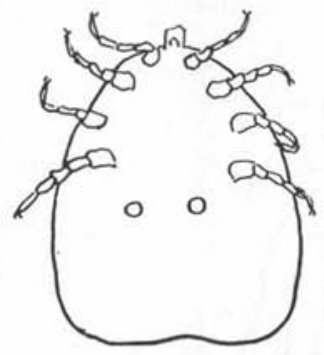
numéraires. Ce spécimen est donc classé dans le groupe II ; il appartiendrait au groupe IV si ces stigmates existaient, mais ce serait peu compatible avec le fait que les écussons ne sont pas dédoublés. Il y aurait donc ici une régression de la monstruosité (fig. 42).

Cet exemplaire a permis aux auteurs que la monstruosité n'est pas héréditaire. Ils ont en effet placé ce mâle avec des femelles normales, et étudié l'évolution de deux des pontes qui ont suivi l'accouplement. Ils ont pu continuer leurs recherches sur six générations

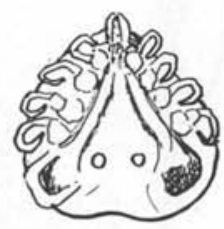

Fig. 42. - Hyalomma manrilanicum. Mâle à deux anus ; les écussons ne sont pas dédoublés. (D'après Sergent et Poncet). successives, comptant 27.710 individus, et aucun n'a montré de malformations. Il serait intéressant de refaire cette expérience en partant d'un mâle et d'une femelle porteurs tous deux de cette anomalie, mais il semble bien que, si la monstruosité double était d'origine génétique, elle aurait dû reparaître au moins de temps à autre.

Type III. - Deux anus, trois stigmates.

Le passage du type III au type IV à quatre stigmates est insensible, car on trouve tous les intermédiaires à stigmates surnuméraires partiellement fusionnés ou étroitement accolés.

Ixodes hexagonus Leach 1815. Brumpt 1934. 
Larve issue d'une femelle gorgée sur Hẻrisson à Rennes. Cette larve présente un dédoublement de la partie postérieure. Au stade nymphal, on voit deux anus et trois stigmates, dont un postérieur. La femelle a les mêmes caractères, le stigmate postérieur est dorsal. On note aussi un début de dédoublement du scutum (fig. 43).

Haemaphysalis punctata. Aboim-Inglez.

Deux adultes. Le stigmate postérieur de l'un deux est très rudimentaire (fig. 26).
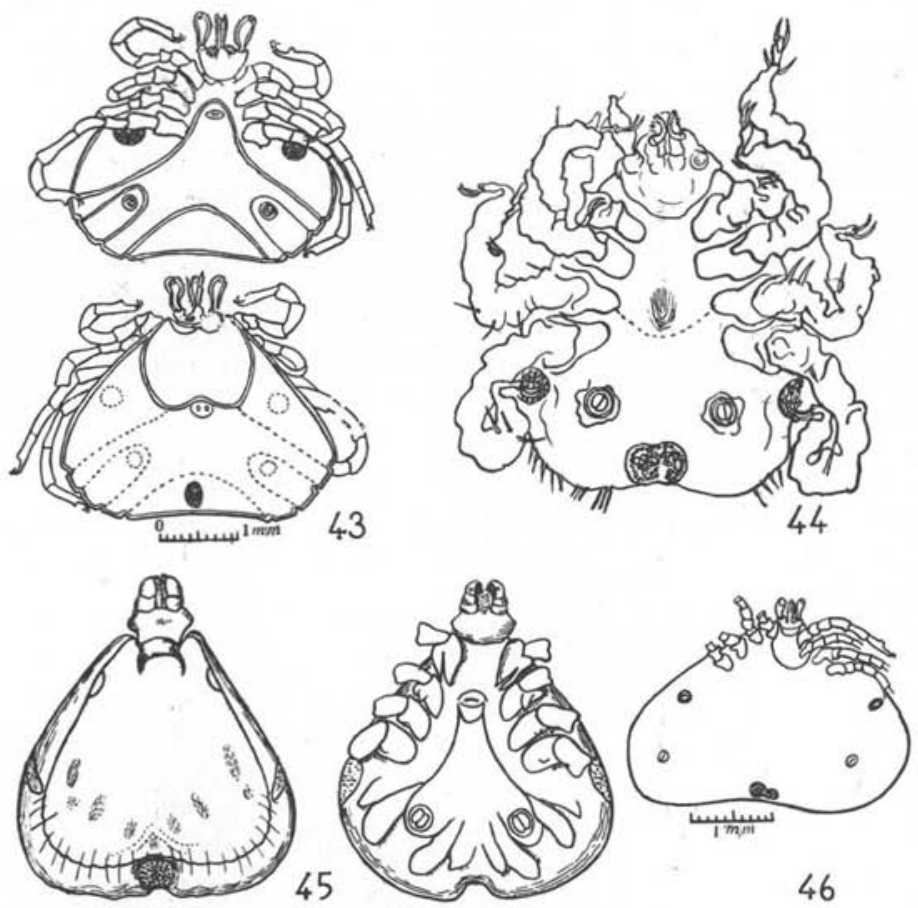

Fig. 43. - Ixodes hexagonus. Nymphe à deux anus et un stigmate surnuméraire dorsal. (D'après E. Brumpt) ; Fig. 44. - Hyalomma mauritanicum. Stigmate surnuméraire à deux lobes. (D'après C. Desportes) ; FIG. 45. - Rhipicephalus sanguineus. Stigmate postérieur dorsal. (D'après Warburton et Nuttall) ; Fig. 46. - Ixodes hexagonus. Nymphe à deux anus et quatre stigmates. (D’après E. Brumpt).

Hybride de Hyalomma anatolicum et de Hyalomma plumbeum. Russie. Pervomaisky 1954.

Mâle avec deux systèmes complets d'écussons. Le stigmate postérieur dorsal est unique, mais possède deux orifices stigmatiques.

Hyalomma dromedarii Koch. Russie. Pervomaisky 1954.

Femelle à deux anus possédant un stigmate postérieur dorsal.

Hyalomma mauritanicum Sénevet 1932. Larve d'un élevage. Desportes 1938 (fig. 44). 
Cette larve cordiforme donne une nymphe qui meurt avant de sortir de la mue larvaire; la partie postérieure intacte montre deux anus et trois stigmates; le stigmate postérieur présente deux lobes.

Rhipicephalus sanguineus Latreille 1804. Gold Coast. Mâle décrit par

Warburton et Nuttall en 1909.

Les écussons sont tous dédoublés et normalement développés, le stigmate postérieur est dorsal (fig. 45).

Type IV. - Deux anus, quatre stigmates.

Les nymphes placées dans ce groupe auraient peut-être donné des adultes du type V.

Ixodes hexagonus Leach 1815. Brumpt 1934.

Larve d'élevage présentant un dédoublement de la partie postérieure. La nymphe possède deux stigmates postérieurs accolés, mais non fusionnés (fig. 46).

Haemaphysalis punctata. Aboim-Inglez.

Une femelle et une nymphe. Stigmates postérieurs accolès (fig. 25-26). Rhipicephalus bursa Canestrini et Fanzago 1877. Brumpt 1934.

Nymphe d'un élevage originaire du Maroc. Les deux stigmates postérieurs sont accolés.

Rhipicephalus sanguineus. Elevage du laboratoire de Parasitologie.

Larve à deux anus qui a donné une nymphe à deux anus et quatre stigmates; les deux stigmates postérieurs sont bien séparés (fig. 47, A et B).
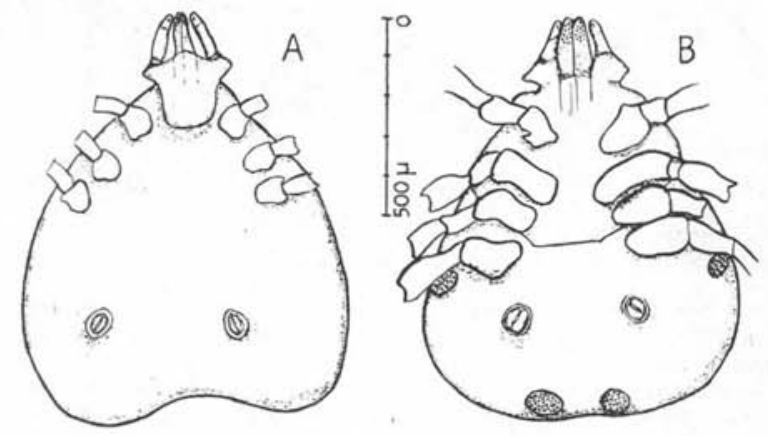

Fig. 47. - Rhipicephalus sanguineus. A, larve à deux anus donnant naissance à $\mathrm{B}$, nymphe à deux anus et quatre stigmates.

Type V. - Deux anus, quatre stigmates, deux orifices génitaux. Haemaphysalis punctata. Aboim-Inglez.

Deux mâles et une femelle. Chez l'un, les deux stigmates postérieurs sont bien séparés, chez les deux autres, ils sont accolés, mais non fusion- 
nés. L'un des deux anus présents à l'état larvaire disparait ensuite chez l'un de ces deux derniers (fig. 25).

Ornithodorus moubata (Murray 1877). Elevage. Robinson 1944.

Nymphe au deuxième stade, qui accentue sa duplication jusqu'au stade femelle : celle-ci possède deux stigmates postérieurs faiblement reliés entre eux, et deux vulves assez petites, inclinées l'une vers l'autre à angle aigu. Cette tique, ayant été fécondée, pond une petite quantité d'œufs, bien qu'ayant refusé de se gorger. Les œufs éclos ont donné des tiques normales (fig. 48).

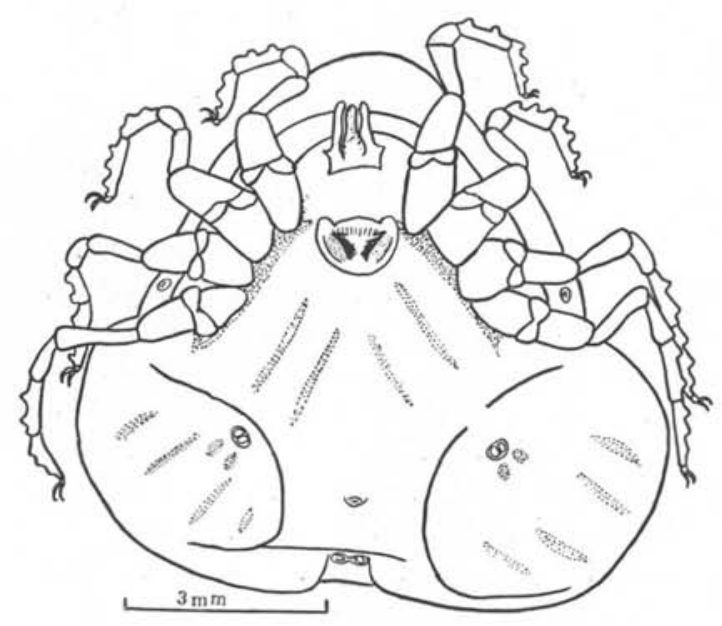

Fig. 48. - Ornithodorus moubata. Femelle à deux anus, quatre stigmates, deux orifices génitaux. (D'après G. Robinson).

Type VI. - Deux anus, quatre stigmates, deux orifices génitaux, deux pattes supplémentaires.

Ixodes ricinus (L. 1746) E. Brumpt 1922, puis 1934.

La larve gorgée présentait une extrémité postérieure fortement dédoublée, avec un seul anus; la nymphe possède deux pattes surnuméraires postérieures, dont chacune est symétrique de la quatrième patte du côté correspondant. Le stade adulte n'a pas été obtenu, mais il aurait certainement eu deux orifices génitaux (fig. 49).

Haemaphysalis punctata. Aboim-Inglez 1942.

Deux mâles et une femelle.

En réalité, un seul exemplaire correspond absolument au type : le deuxième présente une fusion partielle des deux pattes surnuméraires; le troisième n'a qu'un rudiment de patte postérieure médiane au stade larvaire ; cet appendice disparaît ensuite, pour ne plus apparaitre chez l'adulte que sous forme d'un petit disque chitineux (fig. 25). 


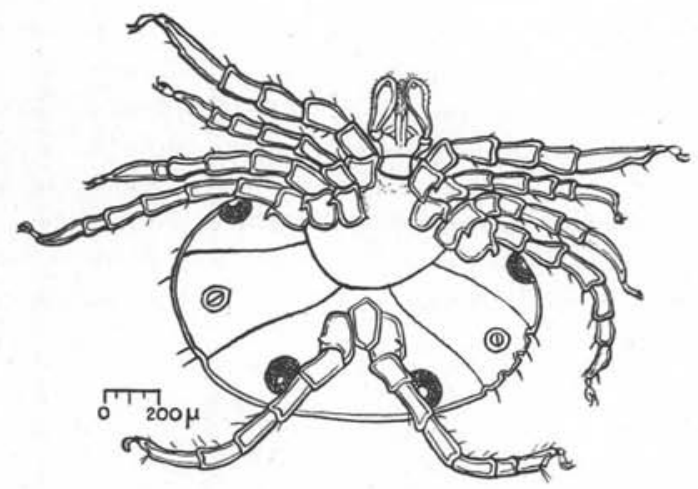

F1G. 49. - lxodes ricinus. Nymphe à deux anus, quatre stigmates et une paire de pattes supplémentaires. (D’après E. Brumpt).

A ces monstres doubles, il faut peut-être ajouter le Hyalomma steineri enigkianum de Schulze (1950), qui présente des duplications d'organes différant beaucoup de ce que nous avons vu jusqu'à présent. Cette femelle possède deux vagins, situés l'un en avant de l'autre sur l'axe médian, le vagin antérieur se terminant en cul-desac. La première patte droite porte, outre l'organe de Haller, une cavité surnuméraire d'où sortent des soies rigides. D'autres anomalies sont encore signalées: fusion des articles 1 et 2 des palpes, absence des chélicères, absence d'une aire poreuse (fig. 50).

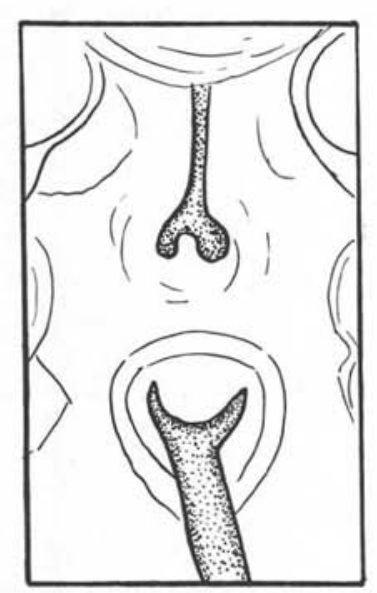

A

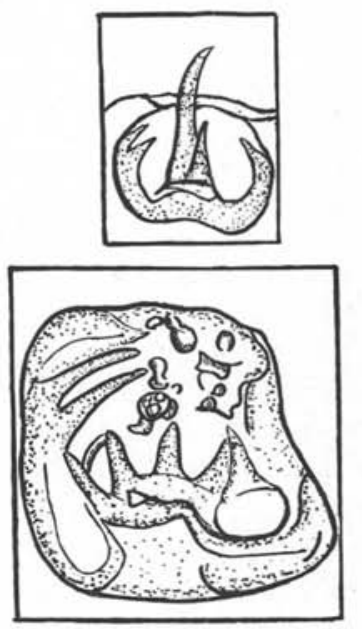

C

Fıg. 50. - Hyalomma steineri enigkianum. A, dédoublement antéro-postérieur du vagin ; $\mathrm{B}$, organe de Haller normal; $\mathrm{C}$, organe de Haller supplémentaire. 
Devant la diversité de ces phénomènes, on peut penser à un traumatisme précoce, survenu durant la vie embryonnaire ou larvaire, plutôt qu'à une anomalie de l'embryogénèse proprement dite.

\section{Origine des monstruosités doubles}

Le monstre double dérive-t-il de la fusion de deux œufs ou d'un dédoublement de l'embryon à un stade plus ou moins précoce ?

Nous savons par les remarquables travaux de Cappe de Baillon que les femelles parthénogénétiques âgées de Carausius morosus pondent assez fréquemment un certain nombre d'œufs composés. Ces œufs proviennent de la réunion de deux ou plusieurs oocytes contenus dans une même chambre ovulaire ; dans la plupart des cas, les ooplasmes fusionnent par suite de la résorption des membranes oocytaires ; la fusion des noyaux n'a pas été observée, mais semble inutile. Chacun d'eux doit contribuer à la formation du blastoderme qui se développe comme un blastoderme normal et forme une ligne germinative unique. La présence sur les œufs d'un double appareil micropylaire influe par sa situation sur l'orientation, et par conséquent sur la morphologie de l'embryon monstrueux. Pendant tout le développement embryonnaire, la régulation de forme intervient pour rapprocher l'embryon du type normal. Les embryons peuvent être symétriques, asymétriques ou irréguliers suivant l'état des lobes procéphaliques des composants. Ils peuvent présenter en outre des anomalies individuelles telles que des inégalités de développement dans une partie du corps, des atrophies ou des concrescences.

Les larves monstrueuses sont également symétriques, asymétriques ou irrégulières. Chez les larves symétriques, les extrémités céphaliques peuvent être fusionnées, distinctes ou opposées ; chez ces dernières, il y a une paire de pattes prothoraciques surnuméraire; chez les larves à extrémités céphaliques distinctes, il y a souvent une patte prothoracique mixte surnuméraire.

Les larves peuvent également présenter des inégalités de développement, des atrophies et des concrescences. Celles-ci aboutissent à la formation d'un appendice incomplet si les ébauches étaient incomplètes, d'un appendice simple, mais de volume double, si les ébauches étaient complètes, et d'un appendice fusionné à la base, mais double à son extrémité, si les ébauches étaient partiellement fusionnées.

Ce dernier fait a servi de base à von Lengerken pour échafauder sa théorie suivant laquelle la plupart des formations multiples et surnuméraires chez les Insectes proviendraient d'œufs fusionnés. 
Ceci est une interprétation tout à fait excessive et, si certaines de ces formations proviennent effectivement de concrescences, il est loin d'en être ainsi pour toutes les schizomélies, comme nous le verrons plus tard.

La régulation qui a tendu à rapprocher le monstre double de la normale pendant la vie embryonnaire continue son action jusqu'à l'apparition de l'imago qui a généralement un aspect normal, la duplicité originelle n'étant plus représentée que par des particularités internes. Ce sont d'ailleurs les larves les moins monstrueuses qui parviennent jusqu'au stade adulte; les autres ont souvent beaucoup de difficultés à muer et à se nourrir et meurent rapidement.

On ne retrouve absolument pas cette régulation chez les Tiques où, le plus souvent, les acquisitions d'organes surnuméraires sont conservées jusqu'au stade adulte.

On a observé des cas de monstruosités doubles chez d'autres Insectes : Tur (1920) a vu des oocytes jumeaux chez le Hanneton (Melolontha vulgaris $\mathrm{F}$.) et pense que le développement de tels œufs donnerait des monstruosités doubles typiques. Beaucoup d'anomalies céphaliques ont certainement une origine double : non seulement les dédoublements d'extrémités céphaliques, mais aussi l'apparition d'une antenne surnuméraire plus ou moins médiane ou d'yeux surnuméraires, sont l'indice d'une telle origine. Il en a été signalé chez les Lépidoptères et les Diptères. Nous n'insisterons pas sur ces cas qui sont bien différents de ce que nous observons chez les Tiques. Une constatation intéressante est à relever : tous les dédoublements observés sont céphaliques ou thoraciques chez les Phasmes, sauf un cas unique où l'animal entier est double et fusionné, sauf le dernier segment abdominal. Chez les autres Insectes, nous ne relevons également guère que des monstruosités des extrémités céphaliques, sauf dans deux cas :

$1^{\circ}$ celui du Limonius canus (Elateridæ) de Woodworth (1932) ; il s'agit d'une larve au premier stade qui présente un dédoublement des quatre derniers segments de l'abdomen ;

$2^{\circ}$ le deuxième cas est le Xenos bohlsi d'Hoffmann (in Lengerken, 1928). Il s'agit encore ici d'une larve primaire dont l'abdomen est dédoublé symétriquement. Ces monstruosités auraient-elles persisté jusqu'au stade adulte, ou, au contraire, auraient-elles subi une régulation analogue à celle des Phasmes ? Elles sont en tout cas extrêmement rares et n'ont été signalées que chez des larves, ce qui serait en faveur de notre deuxième hypothèse. 
On retrouve encore un dédoublement postérieur chez un Myriapode, Stigmatogaster subtecranos Leach. (Selbie 1913).

Voyons maintenant ce qui se passe chez les Arachnides. Les seules anomalies ayant trait à des duplications d'organes connues chez les Aranéides sont des variations du nombre des yeux, ce qui n'a aucun rapport avec la question qui nous intéresse ici. Chez les Scorpions, au contraire, un certain nombre de monstres doubles ont été signalés. La duplication intéresse soit le post-abdomen seul, comme pour le Centrurus infumatus de Berland, soit aussi les derniers segments de l'abdomen, comme pour l'Euscorpus germanicus de Pavesi ou le Buthacus leptochelys d'Et. Sergent. Les deux premiers sont des individus au premier stade, mais le troisième est un adulte. C'est toujours la partie postérieure du corps qui se dédouble. Ceci est tout à fait remarquable et est, à notre avis, fort important, car si ce phénomène est exceptionnel chez les Insectes, nous le retrouvons constamment chez les Tiques qui ne présentent jamais de dédoublement de la partie antérieure.

Peut-on penser que l'origine de ces duplications est très différente de celles des Insectes et, dans ce cas, qu'elle proviendrait d'un dédoublement de l'ébauche embryonnaire primitivement simple? La division se ferait d'arrière en avant et, plus elle serait profonde, plus le degré de duplication serait important. Les expériences de Seidel (1926-28-29) ont montré qu'une cautérisation de l'œuf de Platycnenis pennipes (Odonates) peut provoquer le dédoublement de certains organes dans la région céphalique ou la région abdominale, suivant qu'elle a été pratiquée au pôle supérieur ou inférieur. Le morcellement à la pince peut également aboutir à la production d'un monstre double dont l'un des composants est encastré dans l'autre. Krauss (1934) réalise des dédoublements de certaines parties du corps en employant une fine aiguille pour diviser l'ébauche embryonnaire chez Tachycines asynamorus.

Ewest (1937) effectue des expériences analogues sur des Coléoptères.

Mais tous les monstres obtenus par ces procédés thermiques ou mécaniques ont un aspect particulier, et Cappe de Baillon (1933) a fait observer les différences profondes existant entre ces animaux et les monstres provenant du développement d'une ébauche double. Dans le second cas, on observe toujours une concordance parfaite due au fait que jamais les composants n'ont été totalement séparés et le corps a une symétrie totale qui ne se retrouve pas chez les monstres descendant d'un œuf simple.

Nous retrouvons cette symétrie chez les Scorpions comme chez les Tiques. Chez les premiers, les deux post-abdomens sont nette- 
ment divergents et séparés dès la base, tandis que chez les Tiques l'abdomen reste unique; il est simplement plus ou moins élargi et ne présente pas de traces de fusion des composants. Par contre, les différents organes sont divisés et leur position est presque toujours symétrique par rapport à un axe médian.

Lengerken (1928) donne un schéma des diverses possibilités d'évolution d'un œuf double. L'auteur suppose que les deux composants de chaque œuf, avec leur couche de plasma déterminant, se trouvent de chaque côté du plan sagittal de l'œuf ; les caractères de l'embryon dépendent de la situation de ses couches dont les axes, orientés de la même manière, sont parallèles ou forment un angle aigu. Dans ce dernier cas, si l'angle formé est dirigé vers le haut, on aura des monstres à double extrémité céphalique ; si l'angle est dirigé vers le bas, on aura des animaux à abdomen dédoublé.

Tous nos exemplaires appartiendraient donc à ce type d'œuf. Cappe de Baillon, toutefois (1929), pense que cette formation serait aussi bien expliquée par l'apport tardif de l'un de ses noyaux à l'ébauche embryonnaire, les cellules retardataires ayant pu évoluer sur place et édifier une région du corps déjà ébauchée dans le composant principal. Cette dernière hypothèse ne nous paraît pas très satisfaisante, du moins en ce qui concerne les Tiques. Il y a en effet peu de chances pour qu'un individu ainsi constitué ait des composants identiques. Vachon et Serfaty (1950) font la même remarque à propos des six Scorpions à double queue connus.

Un fait important est en opposition malgré tout avec la théorie de la duplicité de l'œuf : c'est l'absence totale de gynandromorphes parmi les monstres doubles. Chez les Phasmes, il s'agit d'espèces parthénogénétiques, il ne faut donc pas s'étonner de ne rencontrer que des femelles. Cappe de Baillon a d'ailleurs enregistré l'apparition de quelques mâles provenant d'œufs à double micropyle, et surtout de nombreux intersexués dans la descendance des monstres doubles.

Les monstres doubles observés chez les Tiques ne proviennent pas d'espèces parthénogénétiques (Amblyomma rotundatum est ta seule espèce se reproduisant réellement par parthénogénèse). On pourrait donc s'attendre à la présence d'un certain nombre de gynandromorphes parmi eux, or il n'en est rien. Peut-on supposer qu'il y ait prédominance d'un sexe sur l'autre, entraînant dans ce cas l'apparition d'adultes obligatoirement mâles ou femelles ? Parmi les adultes examinés jusqu’à ce jour, il y a une proportion équivalente des deux sexes. En outre, dans les rares cas où l'on a pu obtenir une descendance d'un monstre double, celle-ci est normale. 
Nous sommes donc amenée à supposer que les monstres doubles chez les Ixodoidea proviennent d'un œuf unique; l'ébauche se divise d'arrière en avant à un stade précoce du développement embryonnaire. Brauer (117) indique qu'une division sagittale de la bandelette germinative peut se faire à n'importe quel niveau.

L'embryologie des Tiques est assez bien connue depuis les travaux de Wagner (1894) et de Bonnet (1907). La segmentation est superficielle; c'est dans la région du futur lobe caudal que se forment les cellules endodermiques. Plus tard, les appendices céphaliques et thoraciques, qui occupaient toute la longueur de l'œuf, se rassemblent au pôle antérieur, pendant que le lobe caudal se résorbe, ainsi que la quatrième paire de patte; à peu près au même moment, le cerveau se condense, sauf la dernière paire de ganglions abdominaux qui reste encore assez longtemps représentée par une longue traînée continue avant de se condenser à son tour. Faut-il voir dans cette formation tardive un fait facilitant la division de l'ébauche dans cette région ? Des coupes histologiques, pratiquées pendant les premiers jours du développement de l'œuf, nous montreraient probablement un lobe caudal dédoublé ; malheureusement de tels monstres sont trop rares pour que l'on puisse pratiquer des coupes systématiques d'œufs à divers stades avec quelque chance de succès ; un tel procédé ne sera possible que si l'on arrive à provoquer artificiellement des dédoublements d'embryons, mais là encore on ne sera pas sûr que le processus soit le même que dans la nature. Aboim-Inglez supposait que l'apparition d'un nombre relativement élevé de monstres doubles dans une même génération d'Haemaphysalis punctata pouvait tenir à des facteurs climatiques : ces animaux avaient en effet été récoltés dans une région montagneuse du Portugal où ils avaient été soumis à des différences de température assez considérables. Nous ne pensons pas que ce soit la véritable cause de la monstruosité double, car, dans des expériences antérieures, nous avions soumis des Tiques à l'action de la chaleur ou du froid pour étudier leur biologie et nous n'avons jamais observé de monstruosités dans ces conditions.

En résumé, nous pensons, en accord avec Vachon et Serfaty (1950), que, pour les Tiques comme pour les Scorpions, le monstre double est le résultat, non de la fusion de deux œufs, mais d'une division précoce de la bandelette germinative. Ces dédoublements doivent se former à un niveau quelconque, mais seuls ceux de la région postérieure peuvent aboutir à la formation d'un embryon viable. 


\section{Asymétries}

Si les asymétries légères sont généralement isolées, des déformations plus marquées sont bien souvent associées à d'autres anomalies, en particulier à des ectromélies qui sont très probablement la cause même de l'asymétrie. Nous séparerons donc ces anomalies en deux parties : les asymétries pures et les asymétries associées.

\section{Asymétries pures}

En 1903, Lounsbury remarque de fréquentes asymétries légères chez Argas persicus. Nuttall et Warburton font la même observation en 1908, et donnent une figure montrant effectivement une asymétrie dans la forme du corps et la disposition des disques sur la cuticule. Delpy, en 1936, signale de nombreuses asymétries dans le genre Hyalomma.

Principaux cas :

Amblyomma hebraeum. Guinée portugaise. Robinson 1920.

Le traumatisme initial est évident : la cuticule porte une grande cicatrice atteignant en avant le scutum. L'œil gauche manque.

Amblyomma hebraeum. Mozambique. T. Santos Dias 1947 (fig, 51).

L'axe de symétrie est courbé vers la droite de près de $30^{\circ}$, le bord de l'écusson manque d'un côté et les dessins sont asymétriques.

Amblyomma tholloni. Mozambique. T. Santos Dias 1947 (fig. 52).

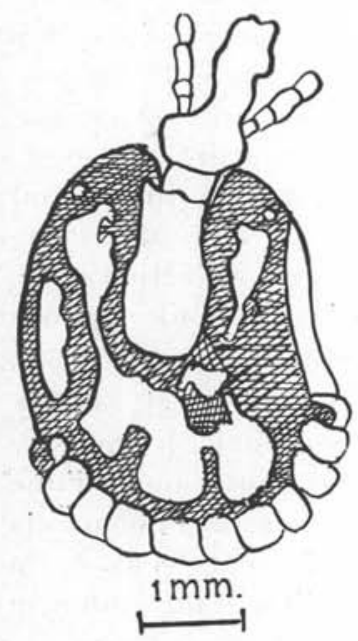

Fic. 51. - Amblyomma hebraeum. Asymétrie du corps et du scutum. (D'après Santos Dias).
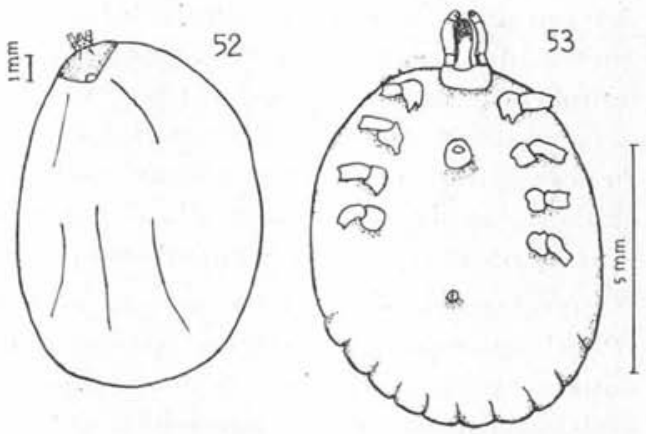

F1G. 52. - Amblyomma tholloni. Femelle asymétrique. (D'après Santos Dias) ; FIg. 53. Amblyomma variegatum. Femelle asymétrique. (Original). 
Amblyomma variegatum. Collection du Laboratoire de Parasitologie.

Le capitulum est incliné vers la gauche (fig. 53).

Ixodes caledonicus. Ecosse. Nuttall 1915 (fig. 54).

Ixodes ricinus. Tchécoslovaquie. Cerny 1957.

Trois exemplaires. Déformations diverses de l'abdomen et du scutum. Haemaphysalis punctata. Tchécoslovaquie. Cerny 1957.

Abdomen resserré à droite au niveau de l'anus.

Hyalomma sp. Indes. Robinson 1920.

Le corps présente une courbure importante et une déformation du scutum. Cette dernière anomalie montre, d'après l'auteur, qu'il ne s'agit

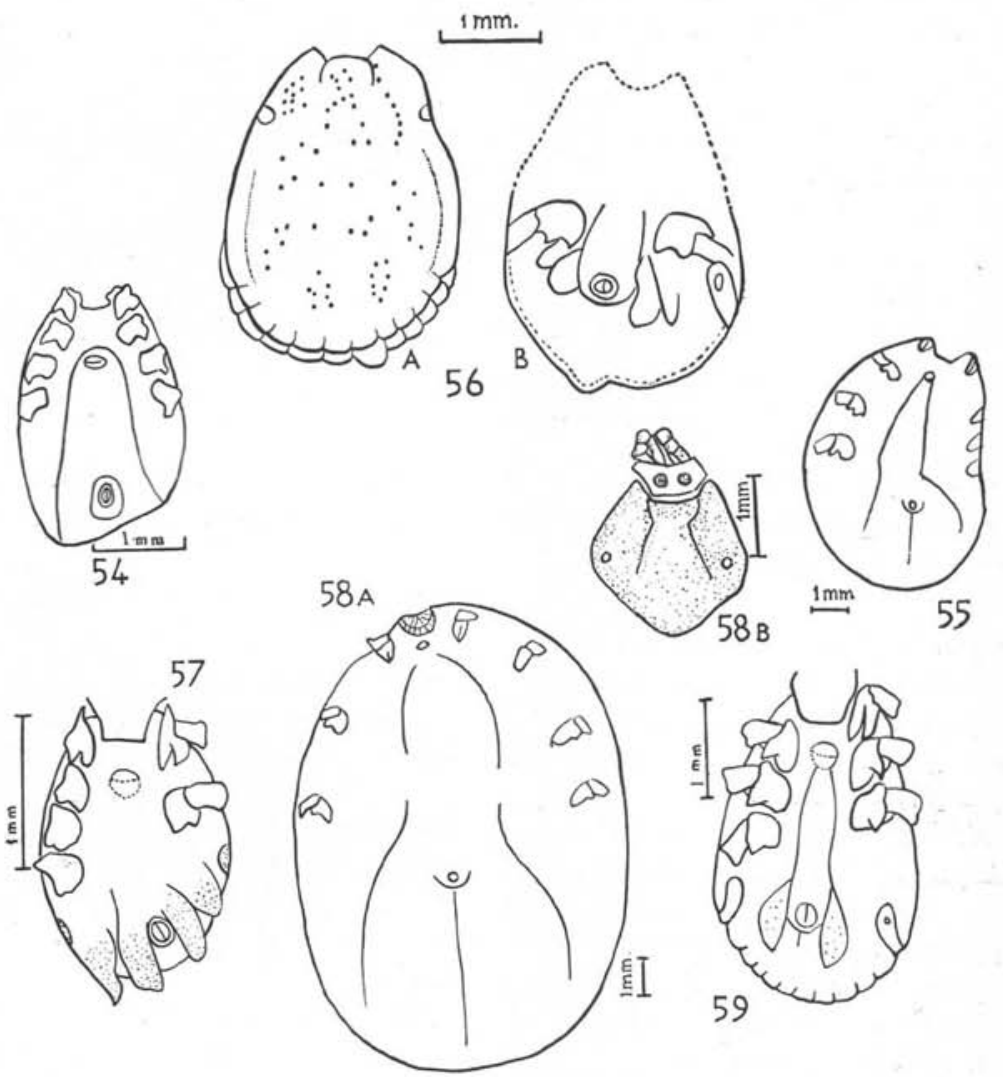

Fic. 54. - Ixodes caledonicus. Asymétrie du bord postérieur. Sillon anal circulaire. (D'après Nuttall); FIg. 55. - Rhipicephalus maculatus. (D'après Santos Dias); Fig. 56. - Rhipicephalus simus. Asymétrie du corps et des écussons. Absence d'un stigmate. (D'après Santos Dias) ; FIG. 57. - Boophilus annulatus. Asymétrie et ectromélie double. (D’après Nuttall); FIG. 58. - A et B, Rhipicephalus evertsi. Asymétrie du corps et du capitulum. Eetromélie de la $2^{\mathrm{e}}$ patte droite. (D'après Santos Dias) ; FIG. 59. - Rhipicephalus pulchellus. Asymétrie et double ectromélie. (D’après Nuttall).

Ann. de Parasitologie, $\mathrm{T}$. XXXIV, $\mathrm{N}^{\circ} 1-2 .-1959$. 
pas d'une simple inégalité de la distension au moment du repas (cause assez fréquemment invoquée).

Hyalomma asiaticum. Russie. Pervomaisky 1954.

Femelle présentant une asymétrie de l'épaule gauche.

Pervomaisky a trouvé dans certains cas de 40 à $65 \%$ d'asymétries; en dehors des malformations expérimentales (amputation de pattes), il cite comme causes l'entassement des larves ou des nymphes pendant le repas. Une pression constante provoque un enfoncement de la chitine qui peut devenir permanent. L'auteur donne quelques exemples.

Hyalomma detritum. Schulze. Russie. Pavlovsky 1940.

Deux exemplaires. Une femelle est complètement arquée vers la gauche, le capitulum se trouvant presque horizontal. L'aspect général est presque celui d'un gynandromorphe, mais la cuticule est entièrement de type femelle.

Rhipicephalus bursa. Larve d'un élevage provenant du Laboratoire de Parasitologie.

Corps dévié vers la gauche, bord postérieur irrégulier.

Rhipicephalus maculatus. Neumann 1901. Mozambique. T. Santos Dias 1947 (fig. 55).

Rhipicephalus simus Koch. Mozambique. T. Santos Dias 1947 (fig. 56).

\section{Asymétries associées}

Ces asymétries sont liées, le plus souvent, à la perte d'une ou plusieurs pattes au stade précédent, que cette perte soit accidentelle ou expérimentale.

Boophilus annulatus (Say). U.S.A. Nuttall 1915 (fig. 57).

Hyalomma anatolicum K. Russie. Pervomaisky 1954.

Après amputation de pattes au stade nymphal, l'auteur obtient un assez grand nombre de tiques asymétriques chez lesquelles les pattes n’ont pas régénéré.

Hyalomma savignyi K. Palestine. Feldman-Muhsam 1950.

Asymétrie du corps liée à l'absence de la $3^{\circ}$ patte gauche ; la nymphe possédait encore un coxa qui a disparu chez l'adulte. Ceci est une preuve supplémentaire de l'origine traumatique des ectromélies.

Rhipicephalus bursa. Russie. Pervomaisky 1954.

Une femelle chez laquelle il reste seulement une patte à gauche; les coxa sont remplacés par de la chitine molle. Le corps est asymétrique. Rhipicephalus evertsi Neumann. Afrique du Sud. T. Santos Dias 1947 (fig. 58).

Rhipicephalus haemaphysaloides (Supino 1877). Indes. Sharif 1930.

Courbure du corps vers la droite, la $4^{\circ}$ patte droite manque.

Rhipicephalus pulchellus (Gerstacker 1873). Afrique occidentale. Nuttall 1915 (fig. 59).

Rhipicephalus sanguineus (Latr.). Indes. Nuttall 1915 (fig. 60). 

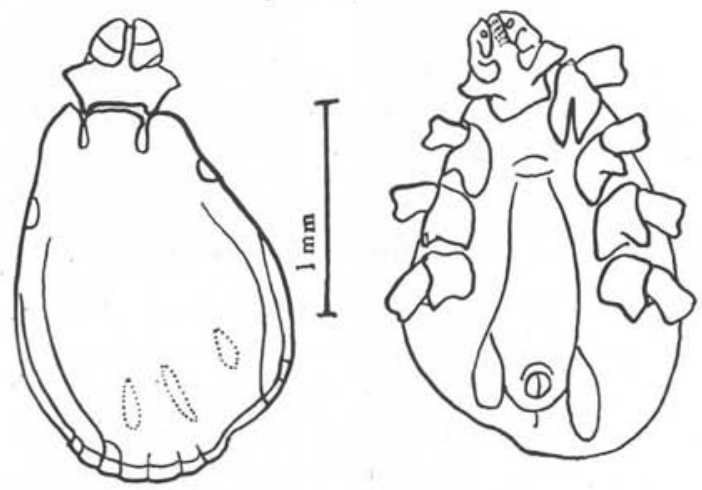

FIG. 60. - Rhipicephalus sanguineus. Asymétrie marquée et ectromélie. (D'après Nuttall).

\section{Origine des asymétries}

Nous voyons que la plupart des exemples cités comportent, outre l'asymétrie du corps, une autre anomalie, telle que l'absence d'une patte ou d'un stigmate. L'absence d'une patte peut évidemment entrainer une déformation de la chitine, mais ces deux caractères ne sont pas forcément liés : c'est ainsi que nous rencontrons des exemplaires auxquels il manque une ou même deux pattes, sans qu'il y ait pour cela la moindre asymétrie.

L'asymétrie seule peut provenir de la façon dont la Tique s'est gorgée, mais elle devrait disparaitre dans ce cas une fois l'animal à jeun. Elle peut également survenir à la suite d'une compression quelconque se produisant au moment de la mue, quand la chitine est encore molle ; celle-ci durcissant assez rapidement, la déformation produite pourra être définitive. C'est notamment ce qui peut se présenter dans des tubes d'élevage où les Tiques sont parfois un peu serrées. Elle peut encore se produire pendant le gorgement si les Tiques sont serrées les unes contre les autres.

Anomalies de la forme générale du corps. - A côté des asymétries proprement dites, nous pouvons ranger des individus ayant une forme anormale par suite d'une distension inégale de la cuticule; l'origine est vraisemblablement la même que celle de certaines asymétries et doit remonter aux précédents repas.

Warburton et Nuttall (1909) signalent qu'un certain nombre de femelles gorgées d'Haemaphysalis punctata sont en forme de poires par suite d'une distension de la partie postérieure plus importante que celle de la partie antérieure. 
Pavlovsky (1940) rencontre la même anomalie chez un Hyalomma sp. qui présente en outre une asymétrie de l'épaule.

Pervomaisky signale de nombreux cas de déformations diverses parmi les hybrides de Hyalomma.

\section{Gigantisme - Nanisme - Variations de taille}

Nous ne pouvons guère parler de gigantisme, ni de nanisme chez les Ixodoidea. En effet, si les variations de taille sont très grandes chez une même espèce (de 1 à 3 dans certains cas), on rencontre tous les degrés entre les dimensions extrêmes. Nous ne parlons pas des femelles gorgées dont les dimensions sont encore plus variables à la suite d'un repas plus ou moins abondant.

C'est parmi les Tiques du genre Rhipicephalus que l'on trouve la plus grande variabilité. Warburton (1912) donne des schémas de tailles extrêmes rencontrées dans un même lot de Tiques provenant de la même femelle, et continuant à être dans des conditions identiques. La longueur varie dans le rapport de 1 à 1,36 pour $R$. simus Koch 1844 , de 1 à 1,62 pour $R$. haemaphysaloides (Supino 1897) et de 1 à 1,48 pour $R$. appendiculatus (Neumann 1901). Cunliffe (1913) examine différents lots de $R$. pulchellus (G. 1873); la longueur des mâles varie de 1 à 1,74 et celle des femelles de 1 à 1,37 . Il étudie un peu plus tard des $R$. sanguineus Latr. dont la taille passe de 1 à 2,08 pour les mâles et de 1 à 1,37 pour les femelles. Ces variations sont dues au mode de nutrition des Tiques aux stades précédents; les spécimens les plus grands proviennent de larves et de nymphes très bien gorgées; les plus petits ont eu un repas incomplet, ayant été retirés de l'hôte avant d'être entièrement gorgés. Nous-même avons enregistré dans un élevage de Dermacentor pictus des variations de 1 à 1,5 chez les mâles et de 1 à 1,25 chez les femelles.

Un fait qui met bien en évidence l'influence de la nutrition sur la taille des individus est que les œufs et les larves à jeun sont de dimensions à peu près invariables; on n'enregistre de différences sensibles que chez la nymphe.

Chez les Ornithodores, dont le nombre de mues nymphales est variable, on pourrait penser que les adultes précoces sont plus petits que les tardifs; des mesures que nous avons effectuées à différentes reprises nous montrent que, si dans certains cas les adultes précoces sont effectivement de tailles plus réduites que les autres, ce n'est nullement un phénomène d'ordre général.

(à suivre). 